\title{
An Integrated Analysis of Capacity Allocation and Patient Scheduling in Presence of Seasonal Walk-ins
}

\begin{abstract}
This study analyzes two decision levels in appointment system design in the context of clinics that face seasonal demand for scheduled and walk-in patients. The macro-level problem addresses access rules dealing with capacity allocation decisions in terms of how many slots to reserve for walk-ins and scheduled patients given fixed daily capacity for the clinic session. The micro-level problem addresses scheduling rules determining the specific time slots for scheduled arrivals. A fully-integrated simulation model is developed where daily demand actualized at the macro level becomes an input to the micro model that simulates the in-clinic dynamics, such as the arrivals of walk-ins and scheduled patients, as well as stochastic service times. The proposed integrated approach is shown to improve decision-making by considering patient lead times (i.e., indirect wait), direct wait times, and clinic overtime as relevant measures of performance. The traditional methods for evaluating appointment system performance are extended to incorporate multiple trade-offs. This allows combining both direct wait and indirect wait that are generally addressed separately due to time scale differences (minutes vs. days). The results confirm the benefits of addressing both decision levels in appointment system design simultaneously. We investigate how environmental factors affect the performance and the choice of appointment systems. The most critical environmental factors emerge as the demand load, seasonality level, and percentage of walk-ins, listed in the decreasing order of importance.
\end{abstract}

Keywords: Operations research in healthcare, appointment scheduling, capacity allocation, simulation, demand seasonality, walk-ins

\section{AUTHORS:}

1. Tugba Cayirli (Corresponding author)

Ozyegin University, Faculty of Business.

Nisantepe Mah. Orman Sok. 34-36, Alemdag, Cekmekoy 34794, Istanbul, Turkey.

Email: tugba.cayirli@ ozyegin.edu.tr Tel: +90 216-564 9516 Fax: +90 216-564 9045.

\section{Pinar Dursun}

Bogaziçi University, Department of Industrial Engineering.

Bebek 34342, Istanbul, Turkey.

Email: pinar.dursun@ boun.edu.tr Tel: +90 (212) 3596407 / 08 Fax: +90 (212) 2651800

\section{Evrim D. Gunes}

Koc University, College of Business Administration and Economics.

Rumeli Feneri Yolu, Sariyer 34450, Istanbul, Turkey.

Email: egunes@ ku.edu.tr Tel: +90 212-338 1639 Fax: +90 212-338 1653. 


\section{An Integrated Analysis of Capacity Allocation and Patient Scheduling in Presence of Seasonal Walk-ins}

\section{INTRODUCTION}

Healthcare costs have increased in the recent years, creating great pressures for healthcare providers to improve operational efficiency while maintaining high service quality. Well-designed appointment systems have the potential to reduce costs by increasing the utilization of expensive resources, and improve service quality by avoiding excessive wait times, both direct and indirect. The indirect wait time, also known as the lead time, indicates the time between the day of request and the day of appointment; and it is an important measure of access to care. The direct wait time indicates how long patients wait in the clinic before they receive the service; and it is one of the major sources of patient dissatisfaction.

In order to provide fair access to care, most clinics admit walk-ins and they are challenged to serve them on the day of arrival. This is further complicated by the fact that demand is usually seasonal with increased rates during flu epidemics or other pandemics. Despite the vast amount of research on appointment scheduling, seasonality of demand in general, and walk-in seasonality in particular, have received limited attention. The problem becomes more challenging when daily capacity is fixed. This is most common given that it is usually not feasible to adjust total capacity or formal operating hours on a daily basis in order to match the seasonal demand patterns.

The main motivation of our research is to investigate how to best accommodate seasonal demand and walk-ins in order to minimize their disruptive effects. To this end, we consider the most challenging scenario where all walk-in demand will be served, using overtime if necessary, when short-term capacity is fixed. Thus it is assumed that two sources of demand compete for a fixed daily capacity measured in number of available slots. These include scheduled patients, who call for appointments in advance, and walk-in patients who arrive without an appointment. The two decisions involve (i) how many slots to reserve for walk-ins and scheduled patients, which is a 'capacity allocation' problem, and (ii) how to assign scheduled patients to specific appointment times, which is a 'patient scheduling' problem. One of the major contributions of this study is to combine these two decisions in the appointment system design, illustrated within the context of seasonal demand for scheduled and walk-in patients. 
We use discrete-event simulation to address the two decision levels in appointment system design using an integrated model that combines the macro and micro levels of analyses. The macro model simulates patients who call for appointments, and their demand is seasonal. The relevant decision variable is the access rule that determines the optimal daily capacity reserved for walk-ins with the remaining capacity allocated to scheduled patients. We test different access rules defined in terms seasonal adjustments made to the daily reservation level for walk-ins, including also the benchmark case with no seasonal adjustment (i.e., daily capacities allocated to two groups of patients are fixed). Next, the micro model simulates the in-clinic aspects on the day of service, including walk-in and scheduled arrivals and stochastic service times. The relevant decision variable at the micro level is the scheduling rule, which sets the specific appointment times for those patients scheduled in advance. This is determined indirectly by setting the open slot positions reserved for walk-ins. A number of scheduling rules are tested using the best policies identified in earlier studies, where the open slot positions remain at the end of a session, evenly-spread, or a mix of both, referred to as the End, Even and EndEven rules. As a result, the combinations of access rules and scheduling rules define our appointment systems. Our simulation model is fully integrated in the sense that the scheduled demand actually assigned to available capacity at the macro level becomes an input to the micro model, allowing for possible empty slots remaining unscheduled. Without such an integration, it is generally assumed that the clinic session starts at full capacity when studies focus only on the micro level of analysis.

The traditional efficient frontier approach used for evaluating system performance is extended for the proposed integrated approach. This incorporates both the macro and micro measures of performance, and therefore results in a more comprehensive evaluation of the best choice of an appointment system. A primary measure at the macro level is the lead time, also known as the "indirect" wait time of patients measured in days. The micro level analysis provides clinic overtime, as well as the "direct" wait time of patients, measured in minutes. Thus both indirect and direct wait times are explicitly included in our assessment of appointment systems using an extended trade-off analysis. This is unlike most of the prior studies that evaluate clinic performance at a single level of analysis, given the difficulty in combining different time scales (i.e., days and minutes). In addition, a secondary measure is used, which is the percentage of patients given appointments within $x$-days, as such policyrelated targets are more common in practice.

In summary, the main contributions of our work are the following: (i) two decision levels in appointment system design are analyzed in an integrated approach in the context of 
seasonal demand, both for scheduled and walk-in patients (ii) a fully-integrated simulation model is used to investigate the best access rules and appointment rules simultaneously, the combination of which defines our appointment systems. As a result, the capacity allocation and scheduling problems are addressed together for a more comprehensive analysis, (iii) all important trade-offs are addressed by including the macro and micro level performance measures, explicitly combining the direct wait and indirect wait that are generally addressed separately due to time scale differences (minutes vs. days) and (iv) environmental factors, including the demand load, walk-in rate and seasonality level, are investigated for their effects on the performance of access rules and scheduling rules.

The rest of the paper is organized as follows. In Section 2, we provide a brief overview of the related literature. Section 3 describes the proposed approach to appointment system design that integrates capacity allocation and patient scheduling problems. The decision variables in terms of access rules and scheduling rules, as well as the relevant cost trade-offs are discussed. In Section 4, we present the methodology, performance measures, simulation model assumptions, and the experimental design. Section 5 discusses the results on the best appointment systems, followed by results on the effects of environmental factors using ANOVA. Finally, Section 6 concludes the paper with a summary of findings, limitations of current work, and some future directions.

\section{LITERATURE REVIEW}

The literature on appointment systems commonly focuses on either access rules at the macro level or scheduling rules at the micro level. First, we discuss studies that investigate access rules for the optimal allocation of available slots (i.e., capacity) to different types of patients. Some common classifications used in the literature include urgent vs. non-urgent, inpatient vs. outpatient demand, and same-day vs. scheduled patients. Patrick and Puterman (2007) analyze an optimal reservation policy that explores the possibility of delaying nonurgent inpatient requests by one day and keeping a pool of outpatient requests available for next day on a short notice. Their simulation results indicate that the introduced flexibility in capacity allocation improves performance significantly in terms of throughput and waiting times. Dobson et al. (2011) analyze optimal carve-out policies for urgent demand and focus on the trade-off between overtime and lead times for routine patients. Urgent patients call for appointment at the beginning of the day, and different scenarios (urgent first, routine first, and hybrid of these extreme cases) are analyzed. Ma et al. (2015) consider the problem of delivering cancer therapy to patients with multi-priority patients, limited specializations of physicians and 
the necessity of patient-physician matching. A simulation and optimization-based framework is used to analyze alternative system configurations with different number of available slots, oncologist specializations, add-on policy, and patient diversion policy. Patrick et al. (2008) model the problem of scheduling multi-priority patient classes to diagnostic imaging resources as a Markov decision process (MDP) and solve the equivalent linear program through approximate dynamic programming. The optimal dynamic policy books a priority class $i$, if it can be booked within a time interval, and diverts patients otherwise. In a subsequent study, Sauré et al. (2012) formulate and solve a radiation therapy-scheduling problem, where the goal is to minimize patients' lead times. The authors expand the multi-priority scheduling model of Patrick et al. (2008) by introducing multi-appointment requirements, multiple session durations, and allowing overtime. Gupta and Wang (2008) develop a dynamic model for the capacity allocation problem for a primary care clinic in the presence of patients' preferences, where the goal is to maximize revenue. Using a MDP approach, the authors determine the optimal carveout level that balances appointment requests for the same day and advanced requests for future appointments, assuming an appointment request of a patient may be denied. A penalty function is included when the clinic cannot meet an appointment request, while in-clinic patient wait times are not tracked. In a subsequent work, Wang and Gupta (2011) extends the work of Gupta and Wang (2008) by proposing an appointment system that uses real time request data to estimate patient preferences. Kolisch and Sickinger (2008) propose an MDP model for the capacity allocation and scheduling problem for two CT-scanners in the context of outpatients, inpatients, and emergency patients. The goal is to maximize revenue where outpatients and inpatients can be denied service at a penalty. Using simulation, the performance of a number of practical capacity allocation rules and scheduling rules are compared with the optimal dynamic solution. Qu et al. (2015) study admission control problem for walk-in patients using an MDP model. They propose a heuristic policy and test its performance under different clinic settings. In this stream of literature, dynamic state information is used to allocate slots to different patient classes, while it is possible to deny some patients.

Second, we discuss the most prominent papers on scheduling rules, where the focus is on the micro level of the problem. The main goal is to optimize the timing of scheduled appointments when the number of appointment slots per day is fixed. A number of papers address strategies to cope with demand variability, including factors such as no-shows, walkins/urgent/same-day patients and/or demand seasonality. Especially, the latter factor is understudied in the literature, even though seasonality of demand in primary care is welldocumented (Forjuoh et al 2001). Rohleder and Klassen (2002) model appointment systems on 
a rolling horizon, where demand load and patterns vary during the week. The authors evaluate different overtime and overbooking combinations, as well as their timing (e.g., delays by 0-2 days) in order to deal with overloads in demand. The results show that good policies change depending on the demand load/pattern, and the choice of performance measures. Both direct and indirect wait times are included in their analysis. Klassen and Rohleder (2004) expand the open slot analysis for urgent clients introduced in Klassen and Rohleder (1996) to a wider set of options in a multi-period scheduling environment. Considering different trade-offs across measures that include direct and indirect wait times for patients, and idle time and overtime for the physician, they conclude that the best slot positions for urgent patients are spread evenly over the day, and a second best option is to spread them evenly over the afternoon. Koeleman and Koole (2012) identify the optimal appointment schedule with non-stationary emergency arrivals via a local search algorithm that starts with some feasible solution, and improves this step-by-step by finding the best solution in its neighborhood. The optimal schedule leaves open slots in the middle or towards the end or distributes them evenly depending on the relative cost of patient's vs. physician's time. Cayirli et al. (2012) introduce an adjustment procedure that explicitly deals with both no-shows and walk-ins, and Cayirli and Yang (2014) extend their rules to patient classification where patients are grouped by the differences in terms of the ratio of no-shows and walk-ins, in addition to the mean and variability of service times. Using simulation optimization, Cayirli and Gunes (2014) investigate scheduling rules to minimize a weighted sum of waiting time, idle time and overtime in presence of seasonal walk-ins. They consider capacity adjustment to seasonality at different levels (monthly, intra-weekly or a combination of both) and show that the combined adjustment is the best strategy. This study extends their analysis by fully integrating the simulation model at the macro and micro levels that allows performance output measured in two time scales (i.e., indirect wait times in days, direct wait times in minutes). As such, access rules are investigated in terms of a scaling factor, called the reservation level coefficient $(\gamma)$, and the explicit modeling of appointment requests allows for possible idle slots in the appointment book at the start of a clinic day. More recently, Borgman et al. (2017) study the appointment scheduling problem for a radiology clinic where unscheduled arrivals have varying due dates, representing their urgency level. Using simulation and a Markov Reward Process, they propose heuristic schedules to minimize waiting time and lateness. Wang et al. (2017) develop a two-stage stochastic optimization model to find the optimal schedules in the presence of walk-ins, to minimize the expected total cost of in-clinic 
wait, idle time and overtime. They find that optimal open slot positions should depend on the arrival pattern of walk-ins.

Kortbeek et al. (2015) is one of the few studies that consider demand seasonality combining macro and micro levels of analysis similar to current work, yet using different performance metrics. The authors develop a methodology to set the capacity allocated to scheduled patients and the timing of appointments, where walk-ins arrive according to a nonstationary process. It is assumed that session length is fixed and there is no overtime in the clinic, while walk-in patients may be deferred to future days by offering appointments. Although they do not make general conclusions, their numerical examples show that open slots should be spread from the middle to the end of the session. This study, along with earlier works by Rohleder and Klassen (2002) and Klassen and Rohleder (2004) is amongst the few that have made combined recommendations using both direct and indirect wait times. In the literature, there are few others that explicitly model two time scales in the context of appointment system design. Creemers and Lambrecht (2010) develop an appointment-driven queueing system model, which is combination of the appointment making queueing system (AMQ) and the service facility queueing system (SFQ). They obtain performance measures such as lead time, direct wait time in the clinic, the probability and the amount of server overtime. Luo et al. (2015) derive performance measures for a queuing model that represents an appointment based service system, consisting of an appointment queue for indirect wait and a service queue for direct wait. Zacharias and Armony (2016) develop an analytical model for indirect and direct wait times, combining the two time scales (days vs. minutes) for an appointment scheduling problem. The authors analyze the joint decision of panel size and the number of daily appointment slots, where all slots are used for scheduled appointments, and show the optimality of open access policy under a set of conditions. The reader is referred to Ahmadi-Javid et al. (2017) for the most recent survey on the optimization studies of outpatient appointment systems.

Finally, there is a related literature on "open access", which has been increasingly popular in primary care (Murray and Tantau 1999, 2000; Murray and Berwick 2003). Research in this area generally focuses on capacity allocation for same-day requests and future appointments (Qu et al. 2007; Qu and Shi 2009, 2011). Using simulation, Kopach et al. (2007) test the effects of four clinic parameters - fraction of open access patients, scheduling horizon for patients on longer-term scheduling, provider care groups, and overbooking on open access implementation. Ratcliffe et al. (2012) consider the heterogeneous no-show probabilities for same-day requests and scheduled appointments, and propose revenue management strategies where overbooking and rejecting patients are possibilities. Balasubramanian et al. (2012) 
analyze the impact of flexibility in a multi-physician setting, where the daily capacity is allocated between pre-scheduled appointments and same-day demand. Their analytical approach assumes the demand is realized instantly while the daily arrival dynamics are ignored. Morikawa and Takahashi (2016) propose a block appointment scheduling method to accommodate walk-ins by assigning them an appointment time at the time of arrival. Their method adds open slots, called a 'box' at the end of each block and walk-ins are allocated to blocks based on the maximum workload allowed. The performance measures are wait times for scheduled and walk-in patients and idle time, while the overtime and lead times are not considered. None of these studies consider the seasonality of demand.

\section{INTEGRATED MODEL OF APPOINTMENT SYSTEM DESIGN}

In this section, we discuss in detail the macro and micro models, when each level is investigated separately with the resulting decision variables and performance measures. This is followed by the proposed integrated model, which combines the macro and micro levels of analyses for a more complete evaluation of appointment systems (see summary in Table 1). All models assume a clinic with a single server, seasonal demand for scheduled appointments and walk-ins, stochastic service times, and punctual patients that always show-up. It is also assumed that all walk-in patients are seen on the day of arrival using overtime, if necessary.

\subsection{Macro Model: Trade-off between Lead Time and Overflow}

The macro model simulates the allocation of two sources of demand, including scheduled patients who call for appointments in advance and walk-in patients who arrive without an appointment. Capacity is measured in number of slots or equivalently, in number of patients, assuming that each patient is assigned to a single slot. The daily target capacity $(T)$ is calculated based on the number of patients to be seen given the session length and the average service time of patients. As an example, if the session length is $7 \frac{1}{2}$ hours per day, and the average service time is 15 minutes, then $T=30$ patients at full capacity. Total capacity is assumed fixed such that $T=R_{t}+N_{t}$, where $R_{t}$ is the reservation level, and $N_{t}$ is the booking limit on day $t$, representing capacities allocated to walk-in and scheduled patients, respectively.

This study proposes adjustments for seasonality through $R_{t}$ in order to avoid excessive overtime in the clinic. This is because although scheduled demand may be postponed to future days when there is no availability, ideally all walk-ins need to be served on the day of their arrival. Therefore, our decision variable on access rules is represented by the reservation level $\left(R_{t}\right)$ for walk-ins. The booking limit is then calculated as $N_{t}=T-R_{t}$ over a planning horizon $H$ 
for day $t=1,2, \ldots, H$. The higher the reserved capacity for walk-ins, the lower the capacity remaining for scheduled patients. As $R_{t}$ increases, the risk of overtime decreases as there are more slots to accommodate walk-ins, yet at the cost of increased lead time for scheduled patients since $N_{t}$ decreases. At the macro level, the number of overflow slots beyond daily capacity is used as an approximation to overtime that can be estimated when the clinic day is observed at the micro level. In order to reflect this trade-off inherent in the macro model, the expected total cost is represented as the sum of the lead time cost and overflow cost:

$$
T C_{\text {Macro }}=c_{L}(L T)+c_{O}(O F)
$$

where $L T$ is the average lead time of scheduled patients in days (calculated as zero if the patient is scheduled on the day of calling). $O F$ is the overflow per day in excess of daily target capacity $T$, measured in discrete number of slots. This estimates the expected number of patients to be seen in overtime. The costs of lead time $c_{L}$ and overtime $c_{O}$ are calculated per day and per slot, respectively. When this trade-off is defined as $\alpha=c_{o} / c_{L}$, taking the cost of lead time as a reference of one, Equation 1 is simplified as $T C_{\text {Macro }}=L T+\alpha(O F)$. This means it is sufficient for the decision maker to estimate a relative valuation without the need for nominal costs. For example, $\alpha=10$ suggests that a one slot increase in overflow per day is 10 times more costly than a one day increase in the lead time of patients. In other words, the clinic is willing to increase average lead times of patients by 10 days to reduce the daily overflow by one slot. In our analysis, $\alpha \geq 1$ is assumed, meaning that the clinic is unlikely to trade-off more than one slot $O F$ for a single day decrease in $L T$. Excluding emergencies, which are handled in separate emergency departments, this assumption will hold true in most ambulatory care services.

\subsection{Micro Model: Trade-off between Wait Time and Overtime}

The micro model simulates the dynamics of the clinic on a service day, including both the scheduled and random walk-in arrivals, as well as the variability in their service times. The relevant decision variable is the scheduling rule, which specifies the appointment times $\left(A_{i}\right)$ for scheduled patients $i=1, \ldots, S_{t}$. In our study, these appointment times are indirectly set through slots remaining from open slot positions reserved for walk-ins. The primary measures at the micro level include the "direct" wait time of patients in the clinic and the overtime per day. (Note that in the rest of the manuscript, we use "wait time" for the direct wait time, whereas we use "lead time" to indicate the indirect wait time). The resulting expected total cost of the system is represented as follows:

$$
T C_{\text {Micro }}=c_{W}\left(W T_{T}\right)+c_{O}(O T)
$$


Table 1. Summary of the Macro, Micro and Integrated Models

\begin{tabular}{|c|c|c|c|}
\hline & MACRO MODEL & MICRO MODEL & INTEGRATED MODEL \\
\hline Problem & $\begin{array}{l}\text { Capacity Allocation } \\
\text { How much daily capacity to allocate for walk-ins } \\
\text { vs. scheduled patients? }\end{array}$ & $\begin{array}{l}\text { Patient Scheduling } \\
\text { What should be the appointment times for } \\
\text { scheduled patients? }\end{array}$ & $\begin{array}{l}\text { Addresses capacity allocation and patient } \\
\text { scheduling problems simultaneously by a full } \\
\text { integration of the macro and micro models. }\end{array}$ \\
\hline $\begin{array}{l}\text { Decision } \\
\text { Variable }\end{array}$ & $\begin{array}{l}\text { Access Rule } \\
\text { Defines daily capacities reserved for walk-ins } \\
\left(\text { reservation level } R_{t}\right) \text { and scheduled patients } \\
\text { (booking limit } N_{t} \text { ) over a planning horizon } H \text {, } \\
\text { given a fixed target capacity }(T) \text { : } \\
\qquad R_{t}=T-N_{t} \quad \text { for day } t=1, \ldots ., H\end{array}$ & $\begin{array}{l}\text { Scheduling Rule } \\
\text { Defines open slot positions for walk-ins based on } \\
\text { reservation level } R_{t} \text {. This consequently } \\
\text { determines appointment times }\left(A_{i}\right) \text { for scheduled } \\
\text { patients } i=1, \ldots, S_{t} \text {. }\end{array}$ & $\begin{array}{l}\text { Appointment System is the combination of } \\
\text { access rules and scheduling rules linking } \\
\text { decisions on: } \\
R_{t}=T-N_{t} \quad \text { for day } t=1, \ldots, H \text {. } \\
\text { and } \\
A_{i} \text { for scheduled patients } i=1, \ldots, S_{t} \text {. }\end{array}$ \\
\hline $\begin{array}{l}\text { Performance } \\
\text { Measures }\end{array}$ & $\begin{array}{l}\text { Total cost of the system calculated as: } \\
T C_{\text {Macro }}=c_{L}(L T)+c_{O}(O F) \\
\text { where } \\
L T \text { is the average lead time of patients (days); } \\
O F \text { is the overflow per day (number of slots); } \\
\alpha=c_{O} / c_{L} \text { is the relative valuation of overtime } \\
\text { and lead time. }\end{array}$ & $\begin{array}{l}\text { Total cost of the system calculated as: } \\
T C_{\text {Micro }}=c w\left(W T_{T}\right)+c o(O T) \\
\text { where } \\
W T_{T} \text { is the total wait time of patients in the } \\
\text { clinic per day (minutes); } \\
O T \text { is the overtime per day (minutes); } \\
\beta=c o / c w \text { is the relative valuation of overtime } \\
\text { and wait time. }\end{array}$ & $\begin{array}{l}\text { - Total cost of the system calculated as: } \\
T C_{I n t}=c_{L}(L T)+c_{W}\left(W T_{T}\right)+c_{O}(O T) \\
\text { where } \\
L T \text { is the average lead time of patients (days); } \\
W T_{T} \text { is the total wait time of patients in the } \\
\text { clinic per day (minutes); } \\
O T \text { is the overtime per day (minutes) } \\
\alpha=c_{O} / c_{L}, \beta=c_{O} / c_{W}, \omega=c_{W} / c_{L} \text { reflect the } \\
\text { trade-offs between each pair of measure, also } \\
\text { including } \omega \text { for the relative valuation of wait } \\
\text { time and lead time. }\end{array}$ \\
\hline
\end{tabular}

${ }^{1}$ Note that the micro measures of $W T_{T}$ and $O T$ originally collected in minutes are converted into $x$-minute number of slots for comparison with the macro model that includes $O F$ measure in discrete units of slots. 
where $W T_{T}$ is the total wait time of all patients in the clinic per day, including wait times for both scheduled and walk-in patients. $O T$ is the overtime beyond the targeted clinic end time per day. Both measures are in continuous time units, originally collected in minutes, and converted into number of slots (e.g., 15 min slots) for ease of comparison with the macro model results. As a result, the cost ratio denoted by $\beta=c_{o} / c_{W}$ indicates the trade-off between the costs of a patient's time and a physician's time. Equation 2 is simplified as $T C_{\text {Micro }}=W T_{T}+\beta(O T)$. For example, $\beta=$ 5 means that a one slot increase in overtime per day is 5 times more costly than a one slot increase in total wait time of patients per day. This means the clinic is willing to increase wait times by 5 slots per day (in total for all patients) in order to reduce daily overtime by one slot. Consistent with the prior literature, we consider $\beta \geq 1$, an assumption that covers most realistic valuations where physician's time is at least as equally valuable as patient's time

\subsection{Integrated Model: Trade-offs between Lead Time, Overtime and Wait Time}

We propose the following total cost function for the integrated model, which incorporates all performance measures and their related cost components, such that:

$$
T C_{\text {Int }}=c_{L}(L T)+c_{W}\left(W T_{T}\right)+c_{O}(O T)
$$

The macro level of the model provides $L T$, whereas the micro level provides $W T_{T}$ and $O T$. The relevant cost parameters include the cost of lead times $c_{L}$ per day, the cost of wait times $c_{W}$ per slot and the cost of overtime $c_{O}$ per slot. First, we replace the overflow measure with the actual overtime obtained from the micro model, such that the true trade-off between lead time and overtime can be analyzed as $T C_{I n t_{-} l}=L T+\alpha(O T)$. Here, $O T$ originally generated in the micro model in minutes is converted into number of slots dividing by $x=15$-minute slots for parallel comparison with the macro model. Note that the resulting $O T$ is in continuous time units of 15minute slots, whereas $O F$ is in discrete units of slots. Second, we include the micro-level trade-off represented by $\beta$ in Equation 2; $T C_{I n t_{-} 2}=W T_{T}+\beta(O T)$. Finally, our integrated analysis incorporates the trade-off between lead time and wait time. This is done by combining $T C_{\text {Int_l }}$ and $T C_{\text {Int_2 }}$, which results in the following equation for the expected total cost:

$$
T C_{\text {Int_3 }}=c_{L}(L T)+c_{W}\left(W T_{T}\right)
$$

where $L T$ is the average lead time of patients, also called the "indirect" wait time, and $W T_{T}$ is the total "direct" wait time of patients in the clinic. $W T_{T}$ measure originally generated in minutes is converted into number of $x$-minute slots. When less capacity is reserved for walk-ins, more remains for scheduled patients who call for appointments in advance. This implies that the lead 
times of patients will decrease, yet at the expense of increased direct wait times. Therefore, it is important to evaluate appointment systems in terms of the trade-offs between direct and indirect wait times of patients in order to balance these conflicting goals.

We denote the new cost ratio as $\omega=c_{W} / c_{L}$ to indicate the cost of a one slot increase in direct wait times relative to a one day increase in lead times, such that Equation 4 is represented as $T C_{\text {Int_3 }}$ $=L T+\omega\left(W T_{T}\right)$. Similar to $\alpha$ and $\beta$, the decision-maker has to provide the relative valuation for $\omega$ without the need for estimating the actual nominal costs. As an example, a choice of $\omega=10$ means the clinic is willing to increase average lead times by 10 days for a one slot reduction in total wait times of all patients per day. We assume no restriction on $\omega$, as the relative costs of lead time and direct wait time can vary widely depending on different specialties and patient groups.

Overall, by using an integrated model, the decision-maker may select the best appointment system, i.e., combination of an access rule and a scheduling rule that will minimize $T C_{\text {Int }}$ in Equation 3, such that all inherent trade-offs $(\alpha, \beta$ and $\omega)$ are completely represented.

\section{METHODOLOGY}

\subsection{Simulation Methodology}

Simulation is chosen as the methodology for analyzing the problem under study, given the flexibility it offers in modeling complex systems. This facilitates addressing both the capacity and scheduling decisions in appointment system design, and overcome the difficulty in modelling nonstationary demand analytically. The simulation model is developed using ARENA software (Version 13.9). Simulation runs are conducted over 50 replications, each of which represents the average of 50 years (i.e., 2500 simulated years for each scenario). A warm-up period of 10 years is excluded from the analysis to reach steady-state results. Pilot runs revealed that for this sample size, performance measures were accurate within $1 \%$ of point estimates at 95 percent confidence level. The common random numbers are used as a variance reduction technique to increase the precision of the estimates when comparing alternative scenarios. Next, we discuss the simulation model assumptions, followed by our integrated simulation model and the related performance measures.

\subsubsection{Simulation Model Assumptions}

A hypothetical clinic is simulated with a session length of $7 \frac{1}{2}$ hours (450 minutes) to represent a clinic that is open from 9:00 am to 4:30 pm. It is modeled as a single-server, singlephase queuing system where there is only one physician and patients receive no service other than the medical consultation. The target number of patients is fixed at $T=30$, corresponding to an 
average service time of 15 minutes with no lunch break. Service times are lognormal with a coefficient of variation of $0.5(\sigma=7.5 \mathrm{~min}$.) and the distribution is assumed identical for walk-ins and scheduled patients. It is assumed that all scheduled patients show up for their appointments and they are strictly punctual. This study sets the no-show probability to zero percent in order to focus on the effect of walk-ins. All walk-ins who arrive before the closing hour are admitted to the clinic and they are served using overtime, if necessary. This assumption is parallel to Cayirli and Gunes (2014), and more recently Morikawa and Takahashi (2016) papers that are empirically based on clinics observed in Turkey and Japan.

In this study, scheduled demand for appointments and walk-in arrivals are modelled as a Poisson process with a seasonal pattern that varies by day of the year $\left(\lambda_{s}^{t}, \lambda_{w}^{t}\right)$. It is assumed that scheduled and walk-in patients follow the same seasonal pattern, in line with Cayirli and Gunes (2014) that report parallel patterns in the empirical data collected. Further details about the formulation of the demand process with seasonality and demand load effects are provided in Appendix 1. In terms of the queue discipline, walk-ins are given lower priority than scheduled patients. As long as there is a scheduled patient ahead in the queue, a walk-in patient will wait. However, if only walk-ins are waiting when the physician becomes idle, then they will be seen on a first-come, first-served basis. It is also assumed that there is no reneging or balking behavior of walk-in patients. First-call, first-appointment (FCFA) rule is used and no patient preferences are considered when assigning calling patients to appointment slots.

\subsubsection{Simulation Model Overview and Performance Measures}

In our fully-integrated simulation model, the macro and micro levels of analyses are combined as summarized in Figure 1. At the macro level, scheduled patients call for appointments, and they are assigned to the day with the first available appointment. The daily requests are generated with a Poisson process where the average number of arrivals are adjusted for seasonal pattern $\left(\lambda_{S}^{t}\right)$ over a one-year planning horizon $(H=240$ days $)$. As discussed in Section 3.1, our macro-level decision variable on access rules determines the reservation level for walk-ins $\left(R_{t}\right)$, which consequently specifies the booking limit for scheduled patients as $N_{t}=T-R_{t}$. Based on the actualized demand, the number of scheduled patients $\left(S_{t}\right)$ are derived for day $t$. When daily assignments are less than the available capacity $\left(S_{t}<N_{t}\right)$, the difference indicates unassigned slots that remain idle in the appointment book. Patients who are assigned to the same day have zero lead times. When patient requests exceed available capacity, and thus are assigned to future days, (positive) lead times are calculated for each patient $i$. Overall, the daily averages computed as $L T$ 
$=\sum_{i}^{S t} L T_{i} / S_{t}$ are averaged across the simulated years for an estimation on average lead times of scheduled patients (in days).

At the micro level of our integrated model, the in-clinic dynamics are simulated on the service day that starts with the actualized number of scheduled patients $\left(S_{t}\right)$ linked from the macro model. As discussed in Section 3.2, our micro-level decision variable on the scheduling rule sets the appointment times $\left(A_{i}\right)$ for scheduled patients. The micro-level performance measures from the simulation model include the in-clinic or direct wait times of patients, and the overtime per day (in minutes). The total wait times are calculated based on all patients served on day $t$, including realized scheduled $\left(S_{t}\right)$ and walk-in patients $\left(U_{t}\right)$, such that $W T_{T}=\sum_{i}^{S t} W T_{i}+\sum_{j}^{U t} W T_{j}$ for $i=$ $1, . ., S_{t}$ and $j=1, . ., U_{t}$. The average wait time (per patient) is then calculated as $W T_{p p}=W T_{T} /\left(S_{t}\right.$ $+U_{t}$ ) for day $t$. These daily values are averaged across the simulated years for obtaining the overall estimations on total and average wait times of patients. Similarly, OT is collected daily to represent the time beyond the targeted clinic end time, and the overall average is calculated across the simulated years.

In sum, our fully-integrated simulation model realistically allows for unassigned slots if the scheduled demand falls short of the daily capacity. Instead, if only the micro model is simulated, then a common assumption is that each day starts with a full schedule $\left(S_{t}=N_{t}\right)$. All relevant measures of performance, including the lead time $(L T)$, wait time $(W T)$ and overtime $(O T)$ are evaluated together for important trade-offs. Finally, the integration allows a more comprehensive analysis on the impact of environmental factors on appointment systems, as well as the interaction effects among access rules and scheduling rules.

Figure 1. Integrated Simulation Model Linking Macro and Micro Levels of Analyses

\begin{tabular}{|c|c|c|}
\hline MACRO LEVEL & & MICRO LEVEL \\
\hline $\begin{array}{l}\text { Simulates the patients' requests } \\
\text { for appointments on each day } t \text {. } \\
\text { The access rule determines the } \\
\text { reservation level for walk-ins }\left(R_{t}\right) \text {, } \\
\text { which specifies the booking limit } \\
\text { for scheduled patients as the } \\
\text { residual from the target capacity, } \\
\text { such that } N_{t}=T-R_{t} \text { for day } t \text {. } \\
\text { Based on the actualized demand, } \\
\text { the number of scheduled patients } \\
\left(S_{t}\right) \text { are derived for day } t \text {. } \\
\text { The macro-level measure is } \\
\text { collected on lead times ( } L T_{i} \text { ) of } \\
\text { scheduled patients } i=1, \ldots . S_{t} \text { on } \\
\text { day } t \text { (measured in days). }\end{array}$ & $\begin{array}{l}\text { Actualized number of } \\
\text { patients assigned for da } \\
\left(S_{t}\right) \text { are linked to the } \\
\text { micro model. }\end{array}$ & $\begin{array}{l}\text { Simulates the service day, where each } \\
\text { day starts with the actualized number } \\
\text { of scheduled patients, } S_{t} \leq N_{t} \text {, where } \\
\text { the difference reflects unassigned } \\
\text { slots. } \\
\text { The scheduling rule determines the } \\
\text { appointment times }\left(A_{i}\right) \text { for } i=1, \ldots S_{t} \text {. } \\
\text { Based on the in-clinic aspects, such as } \\
\text { walk-in arrivals and service times, the } \\
\text { micro-level measures are collected on } \\
\text { wait times }(W T) \text { for all patients, and } \\
\text { overtime }(O T) \text { on day } t \text { (both } \\
\text { measured in minutes originally). }\end{array}$ \\
\hline
\end{tabular}


In our simulation runs, we also collected the overflow beyond daily capacity (in number of slots) in order to evaluate the benefits of integration over the macro model used alone. For this purpose, the number of walk-ins are generated artificially at the macro level $\left(U_{t}\right)$ using a Poisson process, where the average number of arrivals $\left(\lambda_{w}^{t}\right)$ vary daily given the seasonality pattern for day $t$. The overflow is calculated as $O F=\max \left\{0, S_{t}+U_{t}-T\right\}$ for day $t$, and the overall average $(O F)$ is calculated across all simulated years. In addition, a secondary performance measure is collected at the macro level as an important indicator of access to care, namely the percentage of patients seen within $x$-days. In some clinics, seeing the majority of patients within a few weeks could be considered reasonable, whereas others may demand shorter lead times. The "open access" clinics have become increasingly popular, where ideally all patients are to be seen the same day, or at least within -0-2 days (Murray and Tantau 1999, 2000; Murray and Berwick 2003). We evaluate $x=2$ and 10 days to represent clinics that are sensitive to patient access times, albeit with varying thresholds on lead times. The reader is referred to Table 2 for a complete list of performance measures. The micro level measures of $W T_{T}$ and $O T$, originally obtained in minutes from our simulation model output, are converted into number of slots in the main analysis.

\subsection{Experimental Design}

In our simulation experiments, we test alternative appointment systems under a wide range of clinic settings. First, we discuss the environmental factors and their levels chosen for our analysis, followed by a discussion on the decision factors, namely the access rules and scheduling rules, the combinations of which define our appointment systems.

Table 2. Performance Measures

\begin{tabular}{|l|l|}
\hline & Macro-Level Measures \\
\hline$L T$ & Average lead time of patients ${ }^{1}$; also known as the 'indirect wait' (days). \\
\hline$\% L T \leq x$ & Percentage of patients with lead times less than or equal to $x$-days $(x=2,10)$ \\
\hline$O F$ & Overflow per day (number of slots) \\
\hline & Micro-Level Measures \\
\hline$W T_{p p}$ & Average wait time per patient ${ }^{2}$, also known as the 'direct wait' in the clinic (minutes). \\
\hline$W T_{T}$ & Total wait time of patients ${ }^{2}$ in the clinic per day (minutes). \\
\hline$O T$ & Overtime per day (minutes) \\
\hline
\end{tabular}

${ }^{1}$ Relevant for scheduled patients, only. ${ }^{2}$ Including both scheduled and walk-in patients. 


\subsubsection{Environmental Factors}

This study investigates three major factors: the level of seasonality (SEAS), the percentage of walk-ins $\left(P_{w}\right)$ and the demand load $(D L)$. Several studies report that demand varies by the day of the week, the month of the year, as well as the hour of the day, yet this phenomenon is not studied rigorously in the literature (Gupta and Denton 2008). Rohleder and Klassen (2002) investigate weekly seasonality at three different patterns. In this study, we use seasonality indices tested in Cayirli and Gunes (2014) based on the empirical data collected from various public and private clinics. The authors model demand seasonality at three levels, including the monthly, intraweekly, and intra-day seasonalities, which reflect the demand variations by the month of the year, the day of the week, and the hour of the day, respectively. Their findings indicate that the intraday seasonality has relatively less impact on the performance of appointment systems, and therefore, in current study we model demand seasonality as the combination of monthly and intraweekly patterns. The arrival rate is represented as $\lambda^{t}=\lambda \cdot I_{i}^{\mathrm{m}} \cdot I_{j}^{\mathrm{w}}$ for day $t=1,2, \ldots, H$, where $I_{i}^{\mathrm{m}}$ and $I_{j}^{\mathrm{w}}$ are the monthly and intra-weekly seasonality indices for month $i$ and day $j$. Assuming 5 work days a week and 4 weeks a month, the planning horizon $H$ is set as one year including 240 days. As discussed in Section 4.1.2, it is presumed that walk-in and scheduled patients follow the same seasonality pattern with arrival rates denoted as $\lambda_{w}^{t}$ and $\lambda_{s}^{t}$, respectively (See Appendix 1 for detailed formulations).

For the purpose of our analysis, it is not the specific pattern that matters, but rather the coefficient of variation $(C v)$ that defines the different levels of seasonality $(S E A S)$, given that each clinic will face a unique seasonality pattern. This factor is tested at low $(\mathrm{L})$ and high $(\mathrm{H})$ levels, based on the intra-weekly and monthly seasonality indices reported in Cayirli and Gunes (2014), corresponding to overall $C v$-values 0.175 and 0.304 , respectively (Table 3 ). For example, when SEAS is low, the daily seasonality index for a Tuesday in June is calculated as $I_{t}=I_{6}{ }^{\mathrm{m}} \cdot I_{2}{ }^{\mathrm{w}}=0.90$ x $0.95=0.855$ for month $i=6$, and day $j=2$.

The second environmental factor is the percentage of walk-ins $\left(P_{w}\right)$ over the total demand. Although most outpatient clinics require patients to schedule appointments, they also need to serve walk-in patients who show up without an appointment. Studies report that walk-ins are usually urgent patients who need immediate attention, and they are more likely to come from lower social classes (Virji 1990). The walk-in rates may range widely depending on the type of specialty (Cayirli and Veral 2003). Cayirli and Gunes (2014) report average walk-in rates ranging between $20 \%$ and $73 \%$ for different specialty clinics in private and public hospitals, respectively. In this 
Table 3. Experimental Design

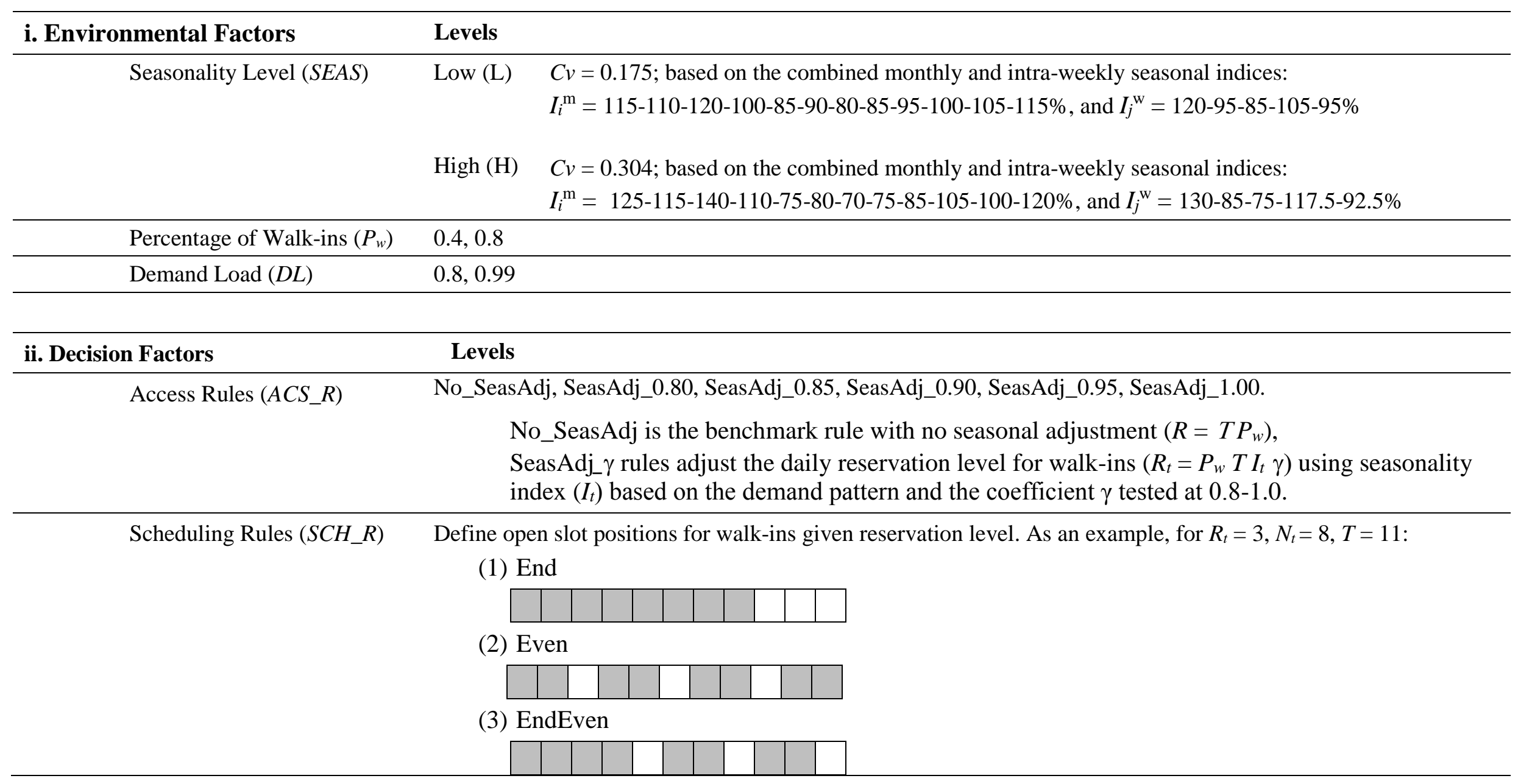


study, we investigate this factor at two levels of $P_{\mathrm{w}}=0.4$ and 0.8 to represent cases where clinics face walk-in rates that are medium and high, under which seasonal adjustments will be preferred.

Finally, the demand load $(D L)$ which is tested in some prior studies (Rohleder and Klassen 2002; Klassen and Rohleder 2004) is hypothesized to be an important factor in the design of appointment systems, and it is investigated at 80 and 99 percent. This reflects system or capacity utilization, and thus may be considered as a decision variable influenced by an appropriate selection of the patient panel size based on a desired service level. As an example, given a capacity of 30 slots per day, an average demand load of 24 patients per day (calls plus walk-ins) would correspond to $80 \%$ utilization and thus $20 \%$ buffer capacity. Therefore, by including the demand load, we indirectly test for the effect of buffer capacity, even though the short-term daily capacity is assumed fixed. $D L=0.99$ represents an extreme scenario, where clinics operate at almost full capacity due to high demand. One may expect that appointment decisions become more critical in clinics that are tight in capacity, compared to those operating with some buffer capacity.

\subsubsection{Decision Factors}

In our simulation experiments, we test eighteen appointment systems that are combinations of six access rules and three scheduling rules. The access rule allocates daily capacities between walk-ins and scheduled patients over a fixed target capacity (See Section 3.1, Table 1). Given the assumption that both sources of demand follow the same seasonal pattern, the daily adjustments on the reservation level $R_{t}$ will impact the booking limit $N_{t}$ in an unparallel manner; i.e., in the opposite direction of seasonality. More specifically, there are two possible cases where the daily demand subject to seasonality is either lower or higher than the yearly average demand. In case of lower demand, $R_{t}$ is reduced in expectation of fewer walkin arrivals, while $N_{t}$ is increased for scheduled patients. This means shorter lead times for scheduled patients, yet at the expense of increased wait times and overtime. The opposite is observed in case of higher demand, when $R_{t}$ is increased and thus $N_{t}$ is decreased, such that the risk of overtime and patient wait times in the clinic is reduced, yet at the expense of longer lead times, especially when demand load is high. To alleviate this effect on lead times, we introduce a coefficient $\gamma<1$ for further investigation. Therefore, our decision variable on access rules is the reservation level adjusted daily over a one-year horizon parallel to the demand pattern using the seasonality index $\left(I_{t}\right)$ and the coefficient $\gamma$, represented as:

$$
R_{t}=P_{w} T I_{t} \gamma \quad \text { for day } t=1,2, \ldots, H
$$


where $I_{t}$ is based on the monthly and intra-weekly seasonality patterns, as discussed and illustrated in Section 4.2.1. Five access rules are tested, denoted as SeasAdj_ $\gamma$ using $\gamma=0.80,0.85,0.90,0.95$ and 1.00 . When no seasonal adjustments are made, then the reservation level is fixed over the entire planning horizon such that $R=P_{w} T$. Thus, the allocation for walk-ins is simply based on the average percentage of walk-ins $\left(P_{w}\right)$ over total demand, where $I_{t}=1$ and $\gamma=1$. This access rule is included as a benchmark policy, referred to as No_SeasAdj. Note that we consider static policies only, i.e., the coefficient $\gamma$ does not depend on time or system state.

The following example illustrates how the proposed access rules adjust for seasonality using a coefficient factor $\gamma$. If $T$ is 30 slots and $P_{w}$ is $40 \%$, then the average capacity to allocate is $R=12$ for walk-ins and $N=18$ for scheduled patients. No_SeasAdj uses these average capacities for the entire planning horizon. If we assume that for day $1, I_{1}=1.38$, then the SeasAdj_0.90 rule with $\gamma=0.90$ allocates $R_{1}=12 \times 1.38 \times 0.9=14.90$ or around 15 slots for walk-ins and the remaining $N_{1}=15$ for scheduled patients. This is calculated similarly for the rest of the days in the planning horizon. Overall, the lower the value of $\gamma$, the less the capacity is reserved for walk-ins, and thus the more remains for scheduled patients since $T$ is fixed.

For simplicity, we use a basic individual-block scheduling rule with fixed intervals of 15 minutes that correspond to the average service time ( $T=30$, session length $450 \mathrm{~min}$.). When seasonal walk-ins exist, the question becomes which particular slots to leave open given the expected number of walk-ins. We include some of the heuristic rules tested in Cayirli and Gunes (2014) based on their simulation optimization results on the best open slot positions. The Even rule spreads open slots uniformly over a clinic session, and the End rule leaves all open slots at the end of the session, whereas the EndEven rule leaves open slots starting from the end slot and then evenly-spread towards the middle. Similar rules have also been shown to perform well in prior studies (Klassen and Rohleder 2004; Koeleman and Koole 2012). Table 3 summarizes our experimental design that includes eight clinic settings ( $2 D L \times 2 S E A S \times 2$ $\left.P_{w}\right)$ and eighteen appointment systems $\left(6 A C S \_R \times 3 S C H \_R\right)$ for a total of 144 scenarios.

\section{RESULTS ON APPOINTMENT SYSTEM PERFORMANCE}

We investigate the performance of our proposed appointment systems using the efficient frontier approach that is commonly used in the appointment scheduling literature when conflicting metrics exist (Ho and Lau 1992). The advantage is that it eliminates the need 
to quantify the exact cost values, and this study extends the traditional approach to evaluate several multiple trade-offs, simultaneously.

In the following Section 5.1, we perform the efficient frontier analysis for the proposed integrated model and compare results to the macro model. We analyze the complete trade-offs between lead time, overtime and wait time, as represented through estimates on their relative cost ratios $(\alpha, \beta, \omega)$. Next, in Section 5.2, we apply the integrated total cost function directly to select the best appointment systems under various clinic settings. This is illustrated for two extreme scenarios on the preferences for the desired cost ratios, referred to as the patientcentered and physician-centered clinics.

\subsection{Efficient Frontier Analysis for the Integrated Model}

The integrated model results are analyzed for the trade-offs between lead times, wait times and overtime. This is done sequentially in three steps by plotting the efficient frontiers for each pair of performance measures as subsets of the integrated total cost function in Equation 3. Then the final choice of an appointment system is determined based on the desirable cost ratios $\alpha, \beta$ and $\omega$, respectively.

The efficient frontiers are obtained for the eight environments tested in our simulation experiments. We illustrate results for the "high" environment with $D L=99 \%, S E A S=\mathrm{H}, P_{w}=$ 80\% (Env_1), which represents the most difficult case with short capacity and high demand uncertainty, both in terms of seasonality and walk-ins. Table 4 tabulates the complete numerical results for Env_1 for all combinations of access rules and scheduling rules tested. The output from the integrated model includes the average lead time of patients in days $(L T)$, the overtime per day $(O T)$, and the total wait time for all patients per day $\left(W T_{T}\right)$. The macro results on lead time $(L T)$ and the overflow per day $(O F)$ are also tabulated in Table 4 in order to evaluate the benefits of full-integration (Note that the overtime measure originally in minutes is converted into number of 15-minute slots for direct comparison with the macro model results).

\subsubsection{Trade-off between Lead Time and Overtime $(\alpha)$}

In the integrated model, the trade-off between the lead time of patients and the overtime per day is represented by the cost ratio $\alpha=c_{O} / c_{L}$ in $T C_{I n t_{-} l}=L T+\alpha(O T)$, which is a subset of the integrated total cost function in Equation 3. An advantage of full-integration is that the measure of overflow $(O F)$ is replaced by the actual overtime $(O T)$ obtained from the micro level of the integrated model. It is observed from Table 4 that $O T$ values are higher in comparison to $O F$ values. As an example, for the SeasAdj_1.00 access rule, overtime is around 3 slots, whereas 
overflow is around 2 slots. This is expected since the micro level analysis accounts for the variability in the clinic, including stochastic service times and arrivals, which are not modeled at the macro level.

Figure $2 \mathrm{i}$ is the corresponding efficient frontier for the illustrated Env_1. Access rules are plotted on a two-dimensional coordinate system using $(O T, L T)$. The $\alpha$ values at which each rule becomes the best choice are calculated from the slopes of the line segments joining the adjacent rules on the efficient frontiers. These $\alpha$ values reflect the cut-off points where the preference shifts from one access rule to another. Strictly inferior access rules can be identified as those remaining above the efficient frontier, resulting in both higher lead time and higher overflow.

The $\alpha$ values for the integrated model are on average 104, 31, 16, 8, 1 for the SeasAdj_ $\gamma$ rules with $\gamma=1,0.95,0.9,0.85,0.8$, respectively. These are averaged across the three scheduling rules of Even, EndEven and End (Table 4, column 9). Thus for Env_1, if the cost of one overflow slot is at least (roughly) 100 times more valuable than a single day increase in the average lead time of patients, then the best access rule is SeasAdj_1.00 with $\gamma=1$. Similarly, the best choice is SeasAdj_0.95 for $31 \leq \alpha<104$, SeasAdj_0.90 for $16 \leq \alpha<31$, SeasAdj_0.85 for $8 \leq \alpha<16$ and SeasAdj_0.80 for $1 \leq \alpha<8$. No_SeasAdj is strictly inferior, indicating that seasonal adjustment on the daily capacity improves clinic performance.

Although the specific $\alpha$ ranges on the best choice of access rules vary for each environment, the general pattern of the efficient frontiers is similar for all the environments tested. The best access rules change from upper-left to lower-right in decreasing order of $\gamma$. This means a smaller coefficient $\gamma$ that reserves less capacity for walk-ins results in shorter lead times for scheduled patients, yet at the expense of higher overtime. Overall, we can generalize that in clinics that place a lower valuation on overtime compared to lead times (i.e., as $\alpha$ decreases), the preference shifts to access rules with smaller $\gamma$ values.

When we compare the integrated model results to those obtained from the macro model, we notice different ranges of $\alpha$ values for the access rules. More specifically, in the macro model the shifts occur at $\alpha$ values 52, 24, 14, 7, 1 for the SeasAdj_ $\gamma$ rules with $\gamma=1,0.95,0.9$, $0.85,0.8$, respectively (Table 4 , column 8). If the clinic prefers $\alpha=15$, then the integrated model suggests SeasAdj_0.85, whereas the macro model suggests SeasAdj_0.90. Thus different access rules may be preferable for a given $\alpha$ depending on the model used, yet the results converge for smaller $\alpha$. We can conclude that our integrated approach provides a more 
Table 4. Comparison of Integrated Model vs. Macro Model Results for Env_1: $D L=99 \%, S E A S=\mathrm{H}$, and $P_{w}=80 \%$

\begin{tabular}{|c|c|c|c|c|c|c|c|c|c|c|}
\hline Access & Scheduling & $\begin{array}{c}\text { Lead Time } \\
\text { (LT) }\end{array}$ & $\begin{array}{c}\text { Overflow } \\
(\text { OF })\end{array}$ & $\begin{array}{l}\text { Overtime } \\
(\text { OT })\end{array}$ & $\begin{array}{l}\text { Wait Time per } \\
\text { patient }\left(W_{p p}\right)\end{array}$ & $\begin{array}{c}\text { Total Wait } \\
\quad\left(W T_{T}\right)\end{array}$ & $\begin{array}{l}\text { MACRO } \\
\text { MODEL }\end{array}$ & \multicolumn{3}{|c|}{$\begin{array}{c}\text { INTEGRATED } \\
\text { MODEL }\end{array}$} \\
\hline Rule & Rule & (days) & (\# slots) & min. (\# slots) & min. (\# slots) & (\#slots) & $\alpha$ & $\left(\alpha_{1}, \alpha_{2}, \alpha_{3}\right)$ & $\left(\boldsymbol{\beta}_{1}, \boldsymbol{\beta}_{2}, \boldsymbol{\beta}_{3}\right)$ & $\left(\omega_{1}, \omega_{2}, \omega_{3}\right)$ \\
\hline SeasAdj_1.00 & $\begin{array}{l}\text { Even } \\
\text { EndEven } \\
\text { End }\end{array}$ & 40.60 & 2.11 & $\begin{array}{l}50.3(3.36) \\
45.1(3.01) \\
44.2(2.95)\end{array}$ & $\begin{array}{l}44.1(2.94) \\
48.9(3.26) \\
57.3(3.82)\end{array}$ & $\begin{array}{r}(88.18) \\
(97.85) \\
(114.55)\end{array}$ & $\alpha \geq 52$ & $\begin{array}{l}\alpha_{1} \geq 96 \\
\alpha_{2} \geq 110 \\
\alpha_{3} \geq 107\end{array}$ & $\begin{array}{l}1 \leq \beta_{1}<28 \\
28 \leq \beta_{2}<269 \\
\beta_{3} \geq 269\end{array}$ & $\begin{array}{l}\omega_{1} \geq 8 \\
\omega_{2} \geq 7 \\
\omega_{3} \geq 20\end{array}$ \\
\hline SeasAdj_0.95 & $\begin{array}{l}\text { Even } \\
\text { EndEven } \\
\text { End }\end{array}$ & 18.68 & 2.53 & $\begin{array}{l}53.8(3.58) \\
48.1(3.21) \\
47.3(3.15) \\
\end{array}$ & $\begin{array}{l}45.5(3.03) \\
50.4(3.36) \\
57.8(3.85) \\
\end{array}$ & $\begin{array}{r}(90.98) \\
(100.81) \\
(115.63)\end{array}$ & $24 \leq \alpha<52$ & $\begin{array}{l}28 \leq \alpha_{1}<96 \\
32 \leq \alpha_{2}<110 \\
33 \leq \alpha_{3}<107\end{array}$ & $\begin{array}{l}1 \leq \beta_{1}<26 \\
26 \leq \beta_{2}<260 \\
\beta_{3} \geq 260\end{array}$ & $\begin{array}{l}2.5 \leq \omega_{1}<8 \\
1.9 \leq \omega_{2}<7 \\
2.0 \leq \omega_{3}<20\end{array}$ \\
\hline SeasAdj_0.90 & $\begin{array}{l}\text { Even } \\
\text { EndEven } \\
\text { End }\end{array}$ & 8.61 & 2.96 & $\begin{array}{l}59.1(3.94) \\
52.8(3.52) \\
51.9(3.46)\end{array}$ & $\begin{array}{l}47.5(3.17) \\
53.1(3.54) \\
60.3(4.02)\end{array}$ & $\begin{array}{r}(95.08) \\
(106.17) \\
(120.63)\end{array}$ & $14 \leq \alpha<24$ & $\begin{array}{l}17 \leq \alpha_{1}<28 \\
16 \leq \alpha_{2}<32 \\
16 \leq \alpha_{3}<33\end{array}$ & $\begin{array}{l}1 \leq \beta_{1}<26 \\
26 \leq \beta_{2}<233 \\
\beta_{3} \geq 233\end{array}$ & $\begin{array}{l}1.4 \leq \omega_{1}<2.5 \\
1.3 \leq \omega_{2}<1.9 \\
1.1 \leq \omega_{3}<2.0\end{array}$ \\
\hline SeasAdj_0.85 & $\begin{array}{l}\text { Even } \\
\text { EndEven } \\
\text { End }\end{array}$ & 2.96 & 3.37 & $\begin{array}{l}64.1(4.27) \\
58.1(3.87) \\
57.2(3.81) \\
\end{array}$ & $\begin{array}{l}49.6(3.31) \\
55.3(3.69) \\
62.9(4.19) \\
\end{array}$ & $\begin{array}{r}(99.21) \\
(110.58) \\
(125.76)\end{array}$ & $7 \leq \alpha<14$ & $\begin{array}{l}9 \leq \alpha_{1}<17 \\
8 \leq \alpha_{2}<16 \\
8 \leq \alpha_{3}<16\end{array}$ & $\begin{array}{l}1 \leq \beta_{1}<29 \\
29 \leq \beta_{2}<238 \\
\beta_{3} \geq 238\end{array}$ & $\begin{array}{l}0.8 \leq \omega_{1}<1.4 \\
0.8 \leq \omega_{2}<1.3 \\
0.7 \leq \omega_{3}<1.1\end{array}$ \\
\hline SeasAdj_0.80 & $\begin{array}{l}\text { Even } \\
\text { EndEven } \\
\text { End }\end{array}$ & 0.58 & 3.69 & $\begin{array}{l}68.0(4.53) \\
62.4(4.16) \\
61.5(4.10)\end{array}$ & $\begin{array}{l}51.1(3.41) \\
56.9(3.79) \\
64.7(4.31)\end{array}$ & $\begin{array}{l}(102.26) \\
(113.69) \\
(129.38)\end{array}$ & $1 \leq \alpha<7$ & $\begin{array}{l}1 \leq \alpha_{1}<9 \\
1 \leq \alpha_{2}<8 \\
1 \leq \alpha_{3}<8\end{array}$ & $\begin{array}{l}1 \leq \beta_{1}<31 \\
31 \leq \beta_{2}<262 \\
\beta_{3} \geq 262\end{array}$ & $\begin{array}{l}\omega_{1}<0.8 \\
\omega_{2}<0.8 \\
\omega_{3}<0.7\end{array}$ \\
\hline No_SeasAdj & $\begin{array}{l}\text { Even } \\
\text { EndEven } \\
\text { End }\end{array}$ & 14.82 & 3.36 & $\begin{array}{l}63.6(4.24) \\
57.9(3.86) \\
56.9(3.79)\end{array}$ & $\begin{array}{l}49.8(3.32) \\
55.1(3.67) \\
62.7(4.18)\end{array}$ & $\begin{array}{r}(99.66) \\
(110.09) \\
(125.41)\end{array}$ & Inferior & Inferior & $\begin{array}{c}1 \leq \beta_{1}<28 \\
28 \leq \beta_{2}<219 \\
\beta_{3} \geq 219\end{array}$ & Inferior \\
\hline
\end{tabular}


Figure 2. Efficient Frontier for the Integrated Model (Env_1: $D L=99 \%, S E A S=\mathrm{H}$, and $P_{w}=80 \%$ ) i. Trade-off between Lead Time and Overtime $(\alpha)$

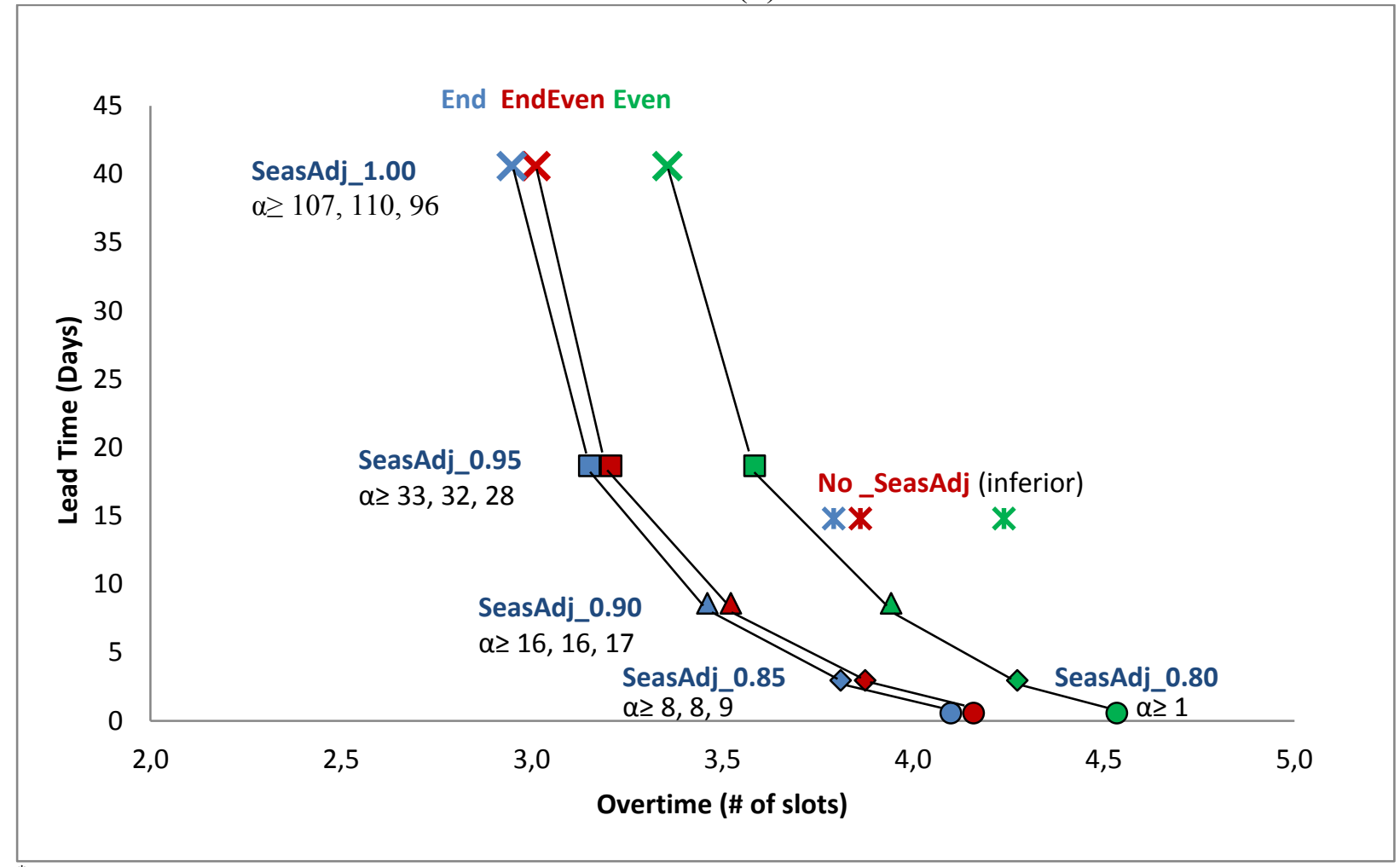

* Cost ratios $(\alpha)$ are listed in order of appearance; such that $\alpha \geq$ End, EndEven, Even

ii. Trade-off between Wait Time and Overtime $(\beta)$

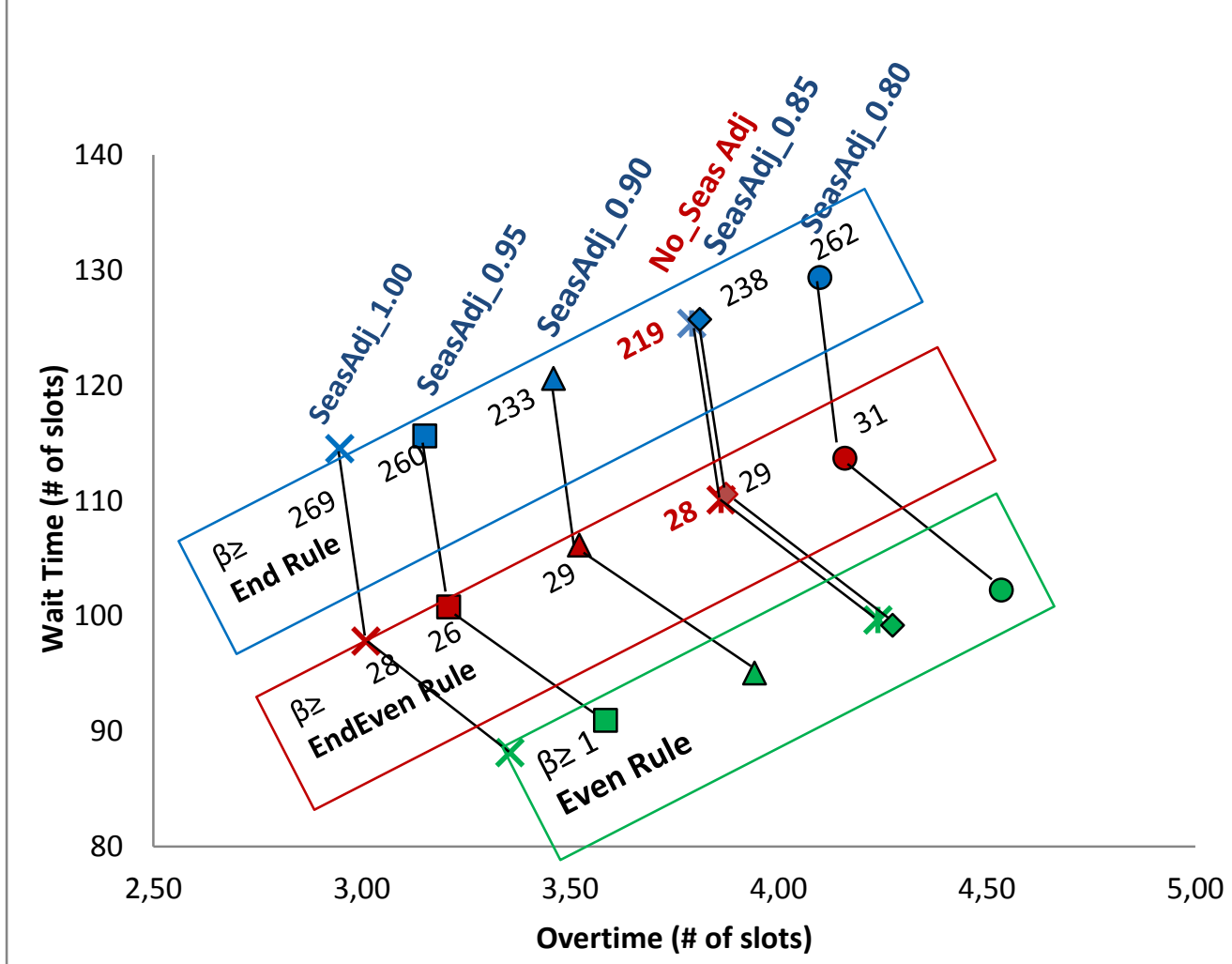


(Figure 2 - Cont'ed)

iii. Trade-off between Lead Time and Wait Time $(\omega)$

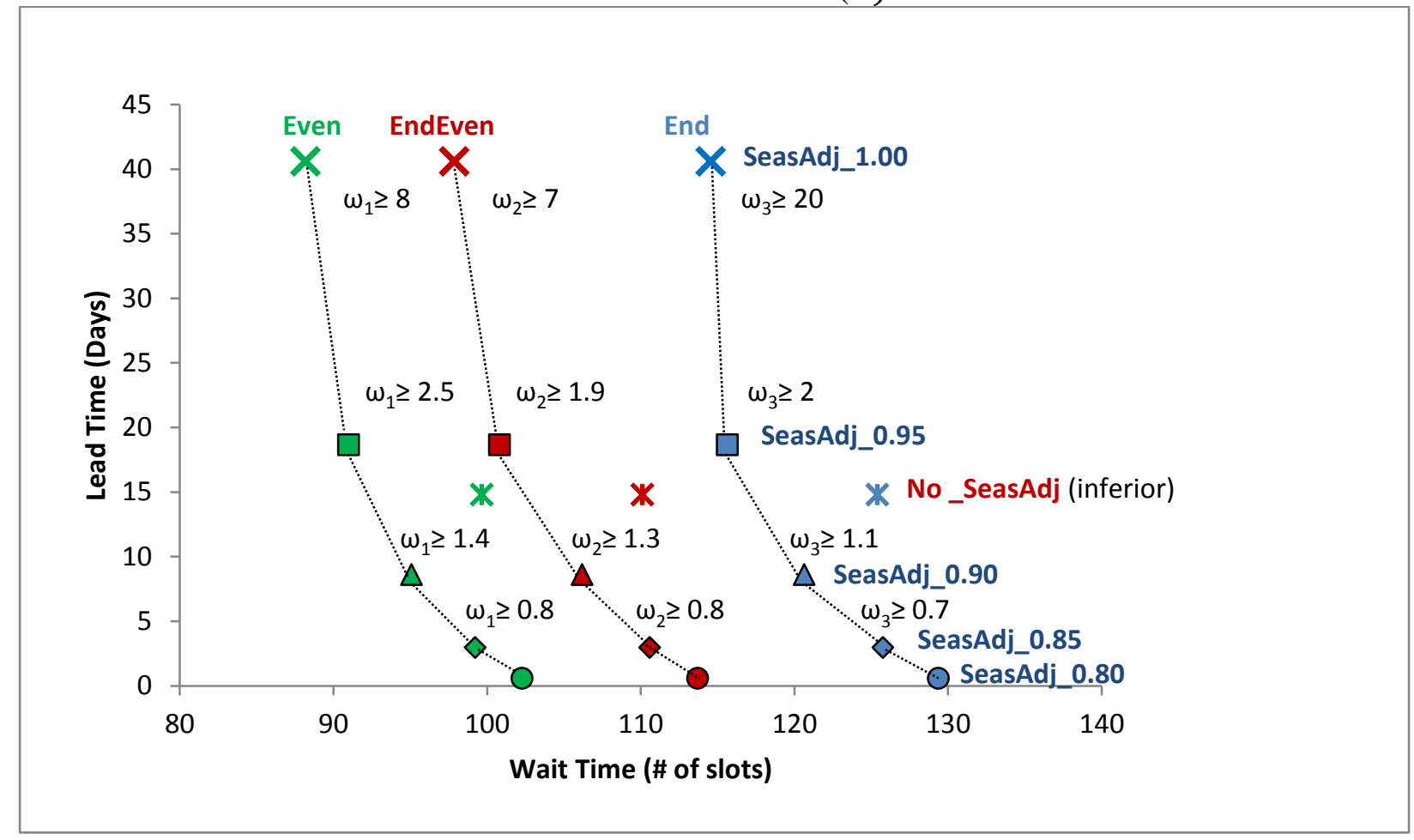

refined choice on the access rules, and these refinements are more pronounced for larger $\alpha$ values.

Moreover, the integrated model captures the details on the scheduling rules, as shown by separate efficient frontiers for each rule in Figure 2i. This is indicated in Table 4 (column 9) by $\alpha_{1}$, $\alpha_{2}$ and $\alpha_{3}$ values as the cut-off points for the Even, EndEven and End rules, respectively. It is observed from the similar $\alpha$ ranges that the choice of an access rule is rather insensitive to the choice of a scheduling rule. In other words, the best access rule may be determined fairly independently of the underlying scheduling rule used.

\subsubsection{Trade-off between Wait Time and Overtime $(\beta)$}

Once the best access rule is determined based on the desired $\alpha$ value as discussed in 5.1.1, the second step is to determine the best scheduling rule based on the cost ratio $\beta=c_{O} / c_{W}$, as represented in $T C_{I n t-2}=W T_{T}+\beta(O T)$, which is a subset of the integrated total cost function in Equation 3. Figure 2ii plots the efficient frontiers for the illustrated "high" Env_1 based on results in Table 4. Each frontier corresponds to an access rule combined with three scheduling rules, namely the Even, EndEven and End rules. The $\beta_{1}, \beta_{2}$ and $\beta_{3}$ values are the cut-off points for switching between two adjacent scheduling rules, as calculated from the slopes on the efficient 
frontiers. For example, if the access rule is SeasAdj_1.00, then the best scheduling rule is End if $\beta$ $\geq 269$, EndEven if $28 \leq \beta<269$, and Even if $1 \leq \beta<28$.

In Figure 2ii, it is observed that for Seas Adj_ $\gamma$ rules with lower $\gamma$, both $W T_{T}$ and $O T$ increase resulting in an upward shift in the efficient frontiers. This is because as $\gamma$ decreases, less capacity is reserved for walk-ins, and this creates higher congestion in the clinic. Another observation is that the slopes joining these scheduling rules are nearly parallel, given the similar $\beta_{1}, \beta_{2}$ and $\beta_{3}$ values across all access rules (Table 4, column 7). This means that the choice for the best scheduling rule remains similar regardless of the underlying access rule. Nevertheless, the choice of an appointment system may change in an integrated approach, when compared to an independent analysis. As an example, let us suppose that the clinic prefers $\alpha=15$ and $\beta=27$. Then, from Table 4, the best access rule is SeasAdj_0.85 and the best scheduling rule is Even. However, for the same $\beta=27$, the best scheduling rule will differ, depending on the $\alpha$-value and the resulting access rule. For example, when $\alpha=20$ and $\beta=27$ the best choice is SeasAdj_0.90 combined with EndEven. Note that this is also the best choice when macro model is used for the access rule, based on $\alpha=15$. As this example illustrates, (i) macro model and integrated model results on the best access rule may differ, and (ii) $\beta$ alone may not provide the optimal scheduling rule, unless the access rule is known. In conclusion, our integrated model provides a more comprehensive and refined approach for choosing the best combination of access and scheduling rules given the desired $\alpha, \beta$ values.

\subsubsection{Trade-off between Wait Time and Lead Time ( $\omega)$}

Most studies on appointment systems consider either the micro or macro level of analysis. This means that the decisions are based on either patients' direct wait times in the clinic, or patients' lead times, also known as the indirect wait times for appointments. By integrating these two levels, our study allows the decision-maker to choose the best appointment system based on the trade-off between these two types of wait, denoted by the cost ratio $\omega=c_{W} / c_{L}$ in $T C_{I n t} 3=L T+\omega\left(W T_{T}\right)$, which is a subset of the integrated total cost function in Equation 3. Figure 2iii shows the efficient frontiers for the same "high" environment (i.e., Env_1). The results on $\omega$-values are provided in Table 4 under the integrated model (column 9), showing the best choices by $\omega_{1}, \omega_{2}$, and $\omega_{3}$ for the Even, EndEven and End rules, respectively. As discussed in Section 3.3, there is no limitation on $\omega$; as it can take any positive value.

In Table 4, the total wait time for all patients $\left(W T_{T}\right)$ is converted into number of slots based on 15-minute appointment slots. As one would expect, the End rule that leaves open slots for walkins at the end of a session results in longest wait times. On the other hand, the Even rule results in 
shortest wait times, and the EndEven rule performs in between. The lead times are only affected by the access rules, and this results in parallel shifts to the right in the order of increasing $W T_{T}$. The results show that $\omega$ ranges usually remain similar across the scheduling rules. One exception is for Seas_Adj_1.00 with $\omega_{1}=8, \omega_{2}=7$, and $\omega_{3}=20$ (Figure 2iii, Table 4). For this access rule, the same decrease in $L T$ results in a lower increase in $W T_{T}$ for the End rule, which starts more

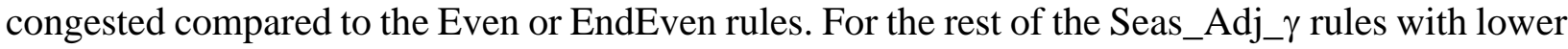
$\gamma<1$, $\omega$ ranges are similar across different scheduling rules, confirming our previous observations that there is little interaction between access rules and scheduling rules.

For the example illustrated in Section 5.1.2 with $\alpha=15$ and $\beta=27$, where the best appointment system is determined as SeasAdj_0.85_Even, the associated $\omega$ range is $0.8 \leq \omega_{2}<1.4$. Thus this appointment system is to be preferred, if the cost of a one slot increase in total wait time of patients per day is approximately equal to the cost of a one day increase in average lead times of patients. This illustrates the benefit of integration where the trade-off on direct and indirect wait times $(\omega)$ can be evaluated in addition to $\alpha$ and $\beta$.

\subsection{Appointment Systems for Patient-Centered and Physician-Centered Clinics}

Although the sequential approach based on the efficient frontiers discussed in Section 5.1 is helpful in illustrating the coexisting trade-offs, an alternative approach is to directly apply the integrated total cost function $T C_{\text {Int }}$ (Equation 3) and choose the best appointment system that minimizes the total cost of the system. However, this requires estimating the nominal cost values on lead times $c_{L}$ per day, the cost of wait times $c_{W}$ per slot and the cost of overtime $c_{O}$ per slot, so that all the inherent trade-offs (i.e., $\alpha, \beta$ and $\omega$ ) are represented.

We illustrate this approach for hypothetical clinics that differ in terms of the desired cost trade-offs, identified as patient-centered and physician-centered clinics to compare the two extremes. Patient-centered clinics favor shorter lead times, despite the increased overtime for the physician. This is represented by cost values of $c_{L}=1$ per day, $c_{W}=1$ per slot, $c_{O}=1.5$ per slot with the resulting cost ratios of $\alpha=1.5, \beta=1.5$ and $\omega=1$. On the other hand, physician-centered clinics prioritize minimizing overtime, at the expense of increasing lead times of patients. The representative cost values are $c_{L}=1$ per day, $c_{W}=1$ per slot, $c_{O}=30$ per slot with the resulting cost ratios of $\alpha=30, \beta=30$ and $\omega=1$ (Later, we use different values of $\omega$; see Figure 3).

We investigate the results for patient-centered and physician-centered clinics under four different clinic settings. The first setting, denoted as Env_1, is the extreme "high" environment illustrated in Section 5.1 with $D L=99 \%, S E A S=\mathrm{H}$, and $P_{w}=0.80$. The second setting keeps demand load the same, whereas both the seasonality level and the walk-in ratio are low (Env_2: 
$\left.D L=99 \%, S E A S=\mathrm{L}, P_{w}=0.40\right)$. These two settings represent clinics with high demand load, or in other words, tight capacity. We also include two additional settings with low demand load combined with high and low levels of seasonality and walk-ins; such that Env_3 represents $D L=$ $80 \%, S E A S=\mathrm{H}, P_{w}=0.80$, and Env_4 represents $D L=80 \%, S E A S=\mathrm{L}, P_{w}=0.40$.

We evaluate eighteen appointment systems for the two clinics under these four extreme settings out of the eight environments tested in our simulation experiments. The results are summarized in Table 5 for each scenario. These scenarios are determined by the clinic setting ( $D L$ $\mathrm{x} P_{w} \mathrm{x}$ Seas $)$, as well as the cost preferences $(\alpha, \beta, \omega)$ indicated by patient vs. physician-centered clinics. The best appointment system(s) for each scenario is (are) listed in the first row of Table 5, where multiple results indicate homogeneous solutions that are not statistically different at alpha $5 \%$ determined by the Bonferroni test. The minimum total cost of the system $\left(T C_{I n t}\right)$ is provided for the best appointment system, along with the percentage improvement over the benchmark policy of No_SeasAdj. Note that the total cost $\left(T C_{\text {Int }}\right)$ measure is defined using "number of slots" as unit of analysis for $O T$ and $W T_{T}$ (see Equation 3). Table 5 tabulates all primary measures of performance, including the average lead time in days $(L T)$, overtime in minutes and in slots $(O T)$, as well as the average total wait time (in slots) and wait time per patient in minutes $\left(W T_{p p}\right)$. In addition, a secondary measure is included on the percentage of patients given appointments within $x$-days $(\% L T \leq x)$ provided for $x=2,10$ days (or 2 weeks).

Some of the important findings are highlighted below:

(i) Seasonal adjustments on daily capacity improve total cost performance in comparison to the benchmark policy of No_SeasAdj. In general, the higher the walk-ins and seasonality levels, the larger the benefits from seasonal adjustments, yet the demand load and cost preferences also impact this relationship. Env_1 with high $S E A S$ and high $P_{w}$, result in largest total cost improvements of around 10\%, whereas the lowest benefits (1-2\%) are observed for Env_2 with low SEAS and low $P_{w}$, and the total cost improvements are similar across patient and physician-centered clinics. Both environments have high demand load $(D L=99 \%)$, representing clinics with tight capacity. This means when demand load is high, the SEAS \& $P w$ combination matters the most in deciding whether it is worthwhile to implement seasonal adjustments. On the other hand, when demand load is low, representing clinics with some buffer capacity, total cost improvements mainly depend on the cost preference. More specifically, in Env_3 and Env_4 with $D L=80 \%$, total cost improvements are around 3\% in patient-centered clinics, yet they increase to $9-10 \%$ in physician-centered clinics, regardless of the differences in SEAS and $P_{w}$ (i.e., low or high). 
Patient-centered clinics prefer access rules with smaller $\gamma$ values that carve-out less capacity for walk-ins to minimize the lead times of patients. For example, in the extreme "high" environment (i.e., Env_1) SeasAdj with $\gamma=0.85$ is best for patient-centered clinics, whereas $\gamma=0.95$ is best for physician-centered clinics. In patient-centered clinics, the average lead time is 3 days with $90.5 \%$ of the scheduled patients seen within 2 weeks. At the other extreme, in physician-centered clinics the lead times are 19 days with only $47 \%$ of the scheduled patients seen within 2 weeks. This improvement in lead times for patientcentered clinics is achieved at the expense of an approximately one slot or 15-minute increase in overtime, when compared to the physician-centered case (i.e., 64.1 minutes vs. 48.1 minutes).

(ii) Two scenarios where the average lead times of patients are less than 1 day correspond to patient-centered clinics with demand load at 80\% (Env_3 and 4). In these scenarios, more than $90 \%$ of the scheduled patients are given appointments within 2 days and $100 \%$ within 2 weeks, representing a level of performance expected from open access clinics. In Env_4 with low walk-ins and low seasonality levels, the performance is further improved in terms of both overtime and patient wait times (i.e., around 15 minutes or one slot; see Table 5). This reflects the additional benefits obtained by controlling for demand uncertainty due to random walk-in arrivals and seasonality in order to improve the in-clinic measures.

(iii) The choice of access rules is sensitive to walk-ins and seasonality levels. As an example, a shift from "high" to "low" levels of walk-ins and seasonality (from Env_1 to 2, or Env_3 to 4) requires an access rule with a higher $\gamma$ value, assuming that the demand load and cost preferences remain the same. This means if the clinic faces higher demand uncertainty due to seasonality and random walk-in arrivals, then reserving less daily capacity for walk-ins provides a better balance in terms of lead times, overtime and wait times for the scenarios tested. This may seem counter-intuitive, yet since the daily target capacity is fixed, reserving less for walk-ins means more slots remain for scheduled patients. In that regard, higher demand uncertainty necessitates a higher buffer capacity for scheduled patients.

(iv) The choice of access rules is less affected by demand load $D L$, when both $S E A S$ and $P_{w}$ are "high". From Table 5, the best $\gamma$ values remain the same across Env_1 and 3, as $D L$ changes from $99 \%$ to $80 \%$ given the same cost preferences. However, under "low" SEAS and $P_{w}$, the effect of demand load is relatively higher. As the $D L$ decreases from $99 \%$ to $80 \%$ (Env_2 to 4), access rules with higher $\gamma$ values are preferred for the same cost preferences. 
Table 5. Best Appointment Systems for Patient vs. Physician-Centered Clinics under Four Selected Environments

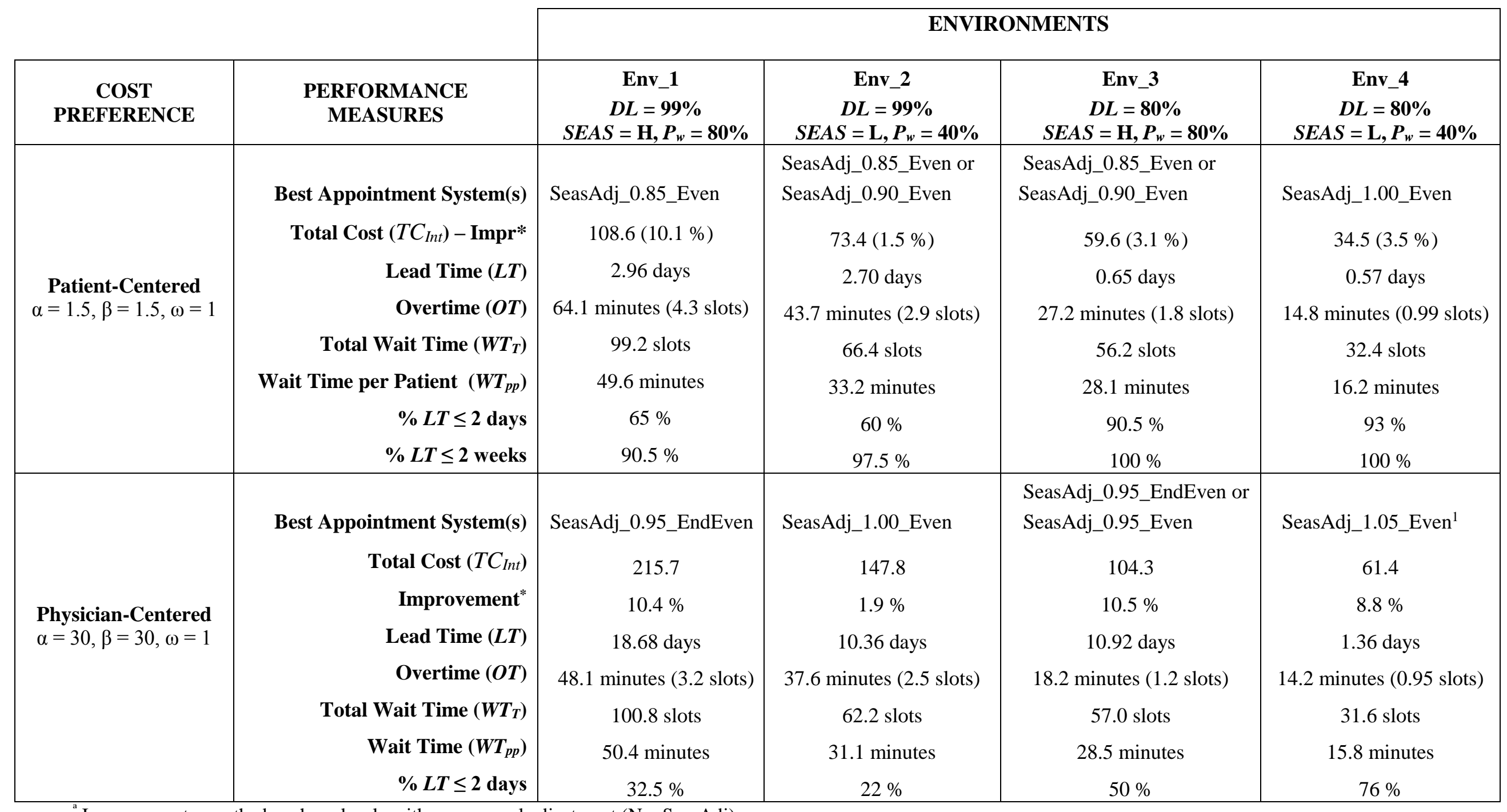

${ }^{a}$ Improvement over the benchmark rule with no seasonal adjustment (No_SeasAdj)

${ }^{1}$ SeasAdj_ $\gamma$ with $\gamma=1.05$ is tested for the extreme "low" Env 4 extending the main runs that included $\gamma \leq 1$. 
(v) In terms of scheduling rules, the Even rule is generally the best choice, except for settings with high walk-ins and high seasonality levels (Env_1 and 3) in physician-centered clinics, where EndEven performs better or at least equally well. This is intuitive since the Even rule spreads open slots uniformly through the session, resulting in lower wait times yet higher overtime in comparison to EndEven. These results confirm the conclusion of Klassen and Rohleder (2004), who found that the Even rule is the best followed by EndEven rule under a different setting. The End rule that leaves all open slots at the end of a clinic session does not perform well under the scenarios tested. This rule becomes the best choice only under very extreme cost ratios $\beta>200$ that are unlikely to be preferred in practice.

Next, we extend the above analysis to different choices of $\omega$ beyond the original value tested $(\omega=1)$, including $\omega=0.5,2,5$ and 10 . Hereby, we fix $\beta$ at 1.5 and 30 for the patient and physician-centered clinics, and evaluate the impact of the choice of $\omega$, which reflects the tradeoffs between direct and indirect wait times.

Figure 3 summarizes results for the "high" environment (i.e., Env\#1). At one extreme, $\omega=$ $c_{W} / c_{L}=0.5$ means that a one slot increase in total $W T_{T}$ in a session is half as costly as a single day increase in $L T$. In other words, the decision-maker is willing to trade-off 0.5 days in $L T$ for a one slot reduction in $W T_{T}$. At the other extreme, $\omega=10$ means the trade-off is between 10 days of $L T$ and one slot in $W T_{T}$. Thus higher $\omega$ values indicate higher preference for prioritizing direct wait times over indirect wait times (i.e., lead times).

Under both patient and physician-centered clinics, the increase in $\omega$ results in access rules with higher $\gamma$, yet the best appointment rules remain unchanged. Higher $\omega$ requires that more capacity is reserved for walk-ins in order to minimize $W T_{p p}$ and $O T$, however this occurs at the expense of higher $L T$. It is observed that lead times are relatively more sensitive to changes in cost parameters, in comparison to the in-clinic measures of overtime and direct wait time. In addition, the shift to appointment systems with higher $\gamma$ occurs slower under physician-centered clinics. This is intuitive given that the higher $\beta=30$ dominates results in physician-centered clinics, whereas the impact of patient-related costs, represented through $\omega$, remains more marginal.

Although omitted for the purpose of brevity, we extend this analysis beyond $\beta=1.5$ and 30 , such that all three cost ratios are included in a single efficient frontier. The reader is referred to Appendix 2 for details on the extended efficient frontier analysis. 
Figure 3. The Impact of Trade-offs Between Direct and Indirect Wait Times (Env\#1)
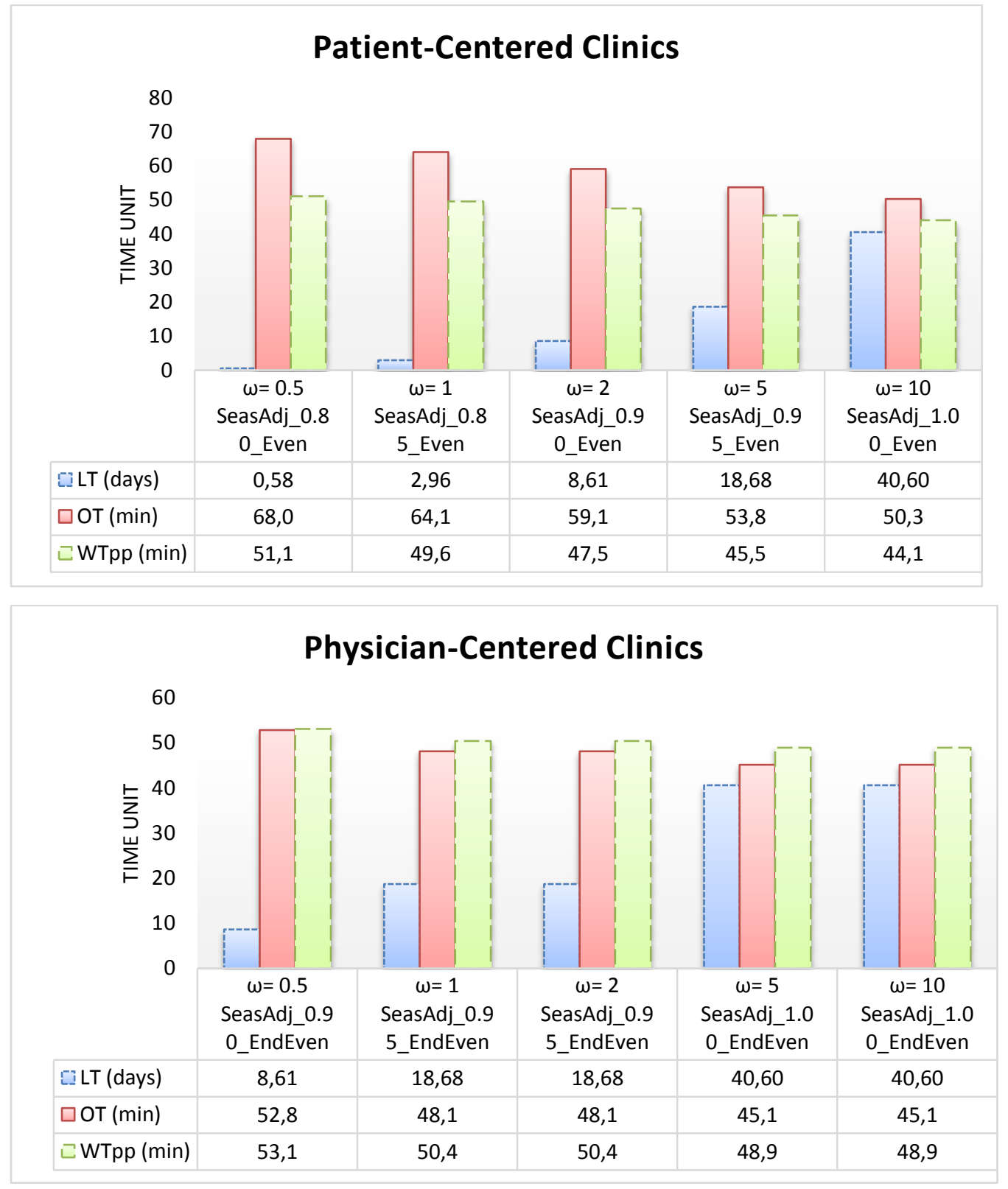

\subsection{Impact of Environmental Factors on Access Rules and Scheduling Rules}

Our analysis in Section 5.2 suggests that there are several interaction effects among the environmental factors and decision factors tested in our study. For this purpose, a full factorial analysis of variance (ANOVA) is conducted to explore the main and interaction effects, extending the analysis beyond the environments investigated in Table 5. The three environmental factors 
include the seasonality level $(S E A S)$, percentage of walk-ins $\left(P_{w}\right)$, and demand load $(D L)$. The decision factors reflecting the two levels in appointment system design include the access rules and scheduling rules, denoted as $A C S \_R$ and $S C H \_R$, respectively. NoSeas_Adj is excluded from further analysis, since this benchmark policy is strictly inferior under all environments as illustrated in the efficient frontier analysis in Section 5.1. Two extreme levels for each environmental factor are included, reducing the number of environments to eight. Table 6 lists results for two total cost performance measures, computed using the original weight parameters used in defining the patient and physician-centered clinics in Section 5.3.

In terms of the main effects, all the environmental and decision factors are significant at alpha 5\%, proving that these factors explain a significant proportion of variation in total cost performance. The major environmental factors are $D L, P_{w}$ and SEAS in the order of decreasing importance as indicated by their decreasing sum of squared values in Table 6. Total cost increases as $D L, S E A S$ and $P_{w}$ increase. These results are in line with the previous literature, noting the importance of demand load/patterns in performance and best scheduling rules (Rohleder and Klassen, 2002). In constrast to Klassen and Rohleder (2004), which conclude that the open slot positions have little impact on the performance, we find that the total cost performance is highly affected by the choice of scheduling rules. This may be due to the fact that much higher ratios for walk-ins are simulated (up to $80 \%$ ) compared to $10 \%$ assumed in prior work. Moreover, our combined analysis on access and scheduling rules also indicates that the decisions on scheduling rules $\left(S C H \_R\right)$, which set the open slot positions (i.e., End, Even, EndEven) are more important than the access rules $\left(A C S \_R\right)$, which determine seasonal adjustment levels through the coefficient $\gamma$ (i.e., Seas_Adj_ $\gamma$ tested at $\gamma=0.8-1.0)$. These observations are valid for both patient and physiciancentered clinics.

From Table 6, all two-way interaction effects are significant at alpha 5\%. Under patientcentered clinics, the most significant two-way interaction occurs between the walk-in probability and access rules $\left(P_{w} \times A C S_{-} R\right)$. As shown in Figure 4i, the performance differences among the access rules are more emphasized under higher $P_{w}=0.80$. On the other hand, for physician-centered clinics, the highest interaction is between the demand load and access rules ( $D L \mathrm{x} A C S \_R$ ). From Figure 4ii, the differences are larger under higher $D L=99 \%$. Although the plot is omitted for the purpose of brevity, similar findings are reported on the $S E A S \times A C S \_R$ interaction. These observations suggest that the value of seasonal adjustments (i.e., $A C S \_R$ ) increase under more 
Figure 4. Two-Way Interaction Plots - Access Rules \& Environmental Factors

i. Total Cost (Patient-Centered): $P_{w} x A C S \_R$

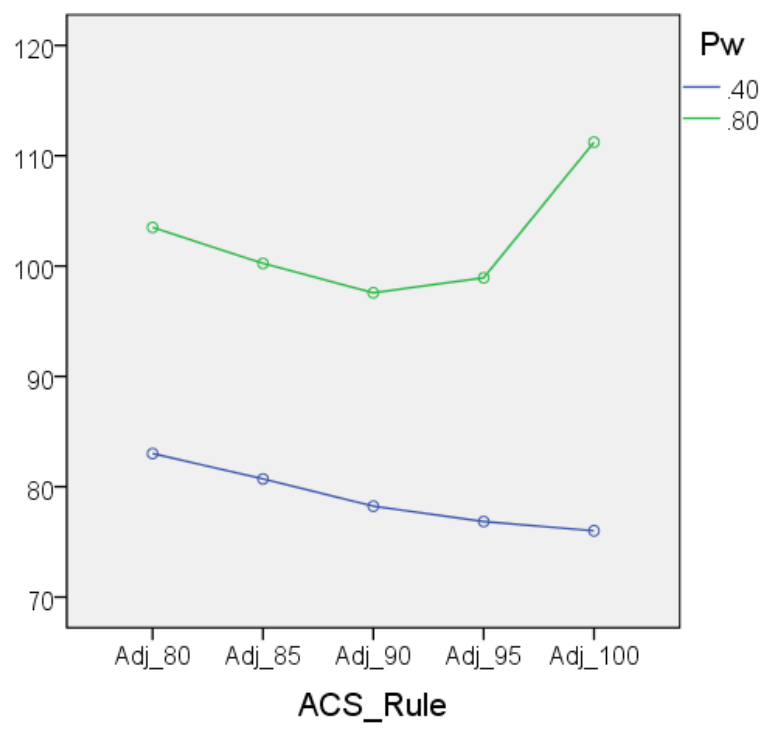

ii. Total Cost (Physician-Centered): $D L x A C S \_R$

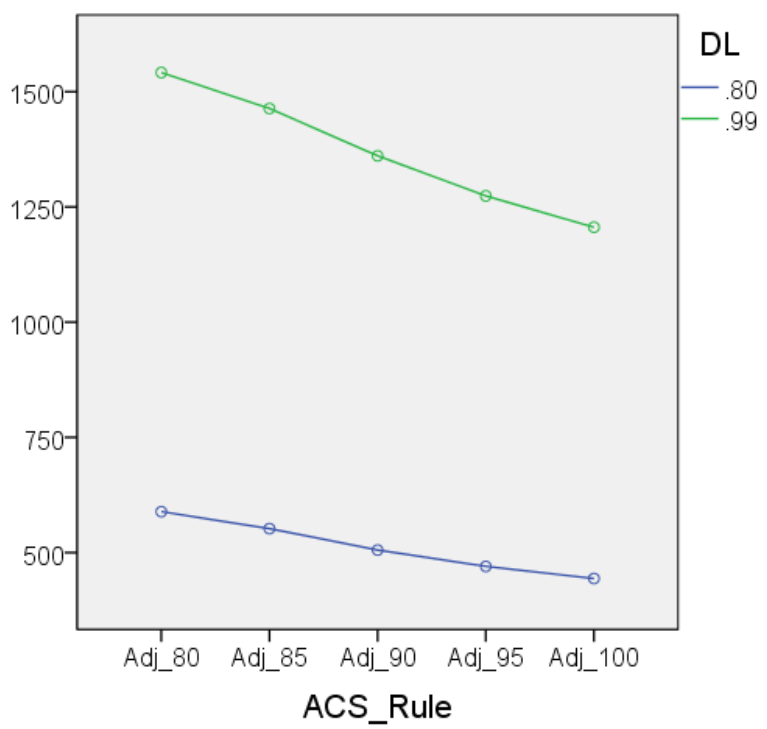

Figure 5. Two-Way Interaction Plots - Access Rules \& Scheduling Rules (ACS_R x SCH_R)

i. Total Cost (Patient-Centered)

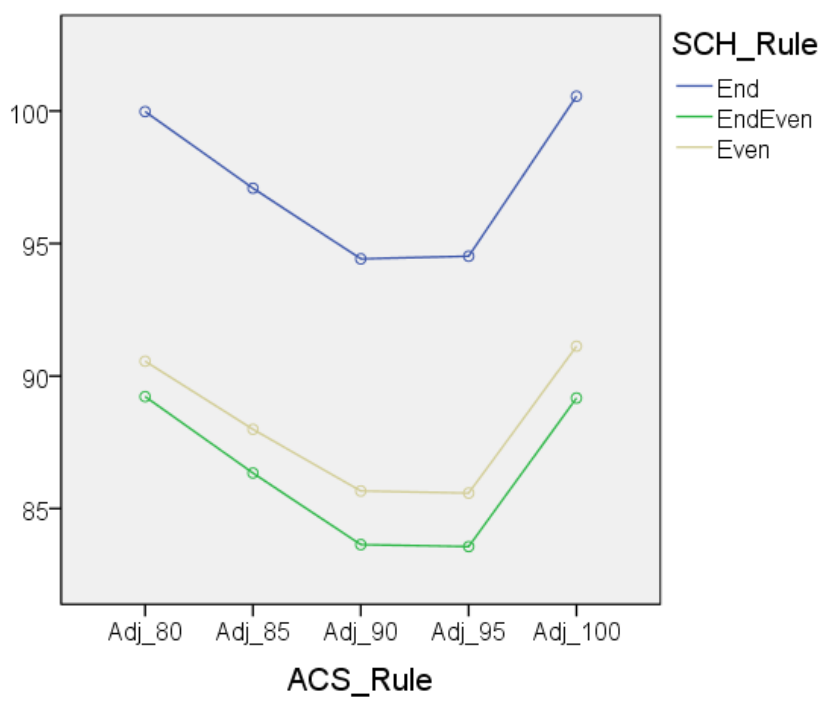

ii. Total Cost (Physician-Centered)

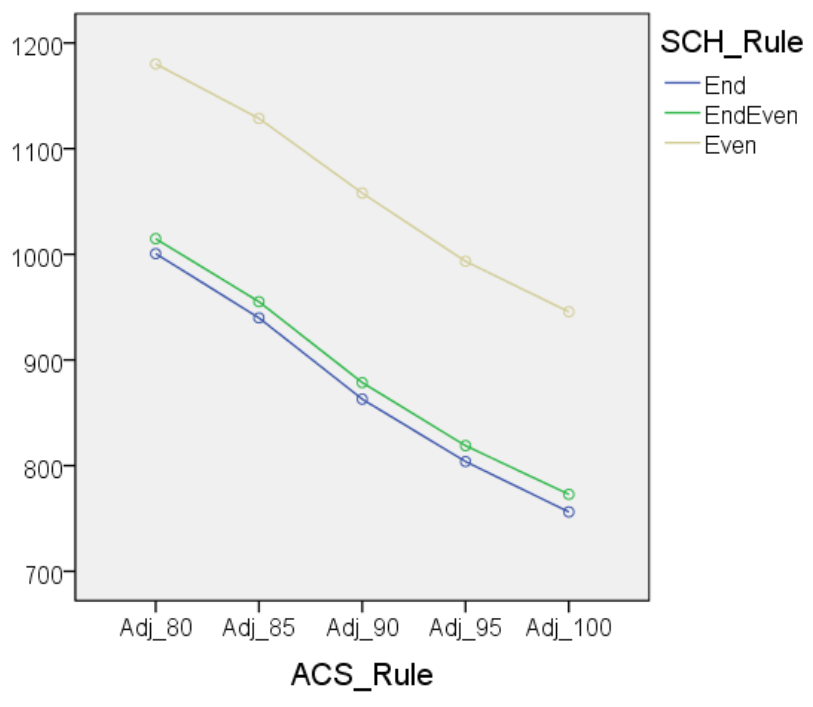


Table 6. Full Factorial ANOVA on Total Cost Measures

i. Patient-Centered Clinics $(\alpha=1.5, \beta=1.5, \omega=1)$

\begin{tabular}{|c|c|c|c|c|}
\hline Source & Sum of $\mathrm{Sq}$ & $\mathrm{df}$ & $\mathrm{F}$ & Sig. \\
\hline Corrected Model & $5074239^{a}$ & 119 & 38309.5 & 0.000 \\
\hline Intercept & 2956881 & 1 & 26565422.5 & 0.000 \\
\hline DL & 4195921 & 1 & 3769729.1 & 0.000 \\
\hline $\mathrm{Pw}$ & 490130 & 1 & 440346.3 & 0.000 \\
\hline SEAS & 122670 & 1 & 110210.0 & 0.000 \\
\hline SCH_R & 82382 & 2 & 37007.3 & 0.000 \\
\hline ACS_R & 22141 & 4 & 4972.9 & 0.000 \\
\hline $\mathrm{DL} * \mathrm{PW}$ & 62138 & 1 & 55826.7 & 0.000 \\
\hline $\mathrm{Pw} * \mathrm{ACS} \_\mathrm{R}$ & 32655 & 4 & 7334.5 & 0.000 \\
\hline $\mathrm{Pw} * \mathrm{SCH} \mathrm{R}$ & 30704 & 2 & 13792.8 & 0.000 \\
\hline $\mathrm{SEAS} * \mathrm{Pw}$ & 25168 & 1 & 22611.8 & 0.000 \\
\hline DL *ACS_R & 1968 & 4 & 442.1 & 0.000 \\
\hline SEAS * ACS_R & 1906 & 4 & 428.0 & 0.000 \\
\hline DL * SEAS & 1878 & 1 & 1687.5 & 0.000 \\
\hline $\mathrm{DL} * \mathrm{SCH} \_\mathrm{R}$ & 474 & 2 & 212.8 & 0.000 \\
\hline ACS_R $* \overline{S C H} \mathrm{R}$ & 80 & 8 & 9.0 & 0.000 \\
\hline SEAS * SCH_R & 30 & 2 & 13.4 & 0.000 \\
\hline $\mathrm{DL} * \mathrm{PW} *$ ACS_R & 1666 & 4 & 374.2 & 0.000 \\
\hline $\mathrm{DL} * \mathrm{SEAS} * \mathrm{PW}$ & 1214 & 1 & 1091.0 & 0.000 \\
\hline DL $*$ SEAS $*$ ACS_R & 253 & 4 & 56.9 & 0.000 \\
\hline $\mathrm{SEAS} * \mathrm{Pw} *$ ACS_R & 239 & 4 & 53.6 & 0.000 \\
\hline $\mathrm{SEAS} * \mathrm{Pw} * \mathrm{SCH} \mathrm{R}$ & 148 & 2 & 66.3 & 0.000 \\
\hline $\mathrm{DL} * \mathrm{Pw} * \mathrm{SCH} \_\mathrm{R}$ & 128 & 2 & 57.6 & 0.000 \\
\hline Pw * ACS_R * SCH_R & 127 & 8 & 14.3 & 0.000 \\
\hline $\mathrm{DL} *$ ACS_R $*$ SCH_R & 53 & 8 & 5.9 & 0.000 \\
\hline SEAS $*$ ACS_R $*$ SCH_R & 5 & 8 & .6 & $0.796^{\mathrm{a}}$ \\
\hline $\mathrm{DL} * \mathrm{SEAS} * \mathrm{SCH} \mathrm{R}$ & 2 & 2 & .7 & $0.492^{a}$ \\
\hline DL $*$ SEAS $*$ Pw $*$ ACS_R & 122 & 4 & 27.5 & 0.000 \\
\hline SEAS $*$ Pw $*$ ACS_R $*$ SCH_R & 18 & 8 & 2.1 & 0.037 \\
\hline $\mathrm{DL} * \mathrm{SEAS} * \mathrm{Pw} * \mathrm{SCH} \mathrm{R}$ & 10 & 2 & 4.6 & 0.010 \\
\hline $\mathrm{DL} * \mathrm{Pw} * \mathrm{ACS} \_\mathrm{R} * \mathrm{SCH} \_\mathrm{R}$ & 4 & 8 & .5 & $0.879^{a}$ \\
\hline $\mathrm{DL} * \mathrm{SEAS} * \mathrm{ACS} \_\mathrm{R} * \mathrm{SCH} \mathrm{R}$ & 3 & 8 & .3 & $0.950^{\mathrm{a}}$ \\
\hline $\mathrm{DL} * \mathrm{SEAS} * \mathrm{PW} * \mathrm{ACS} \_\mathrm{R} * \mathrm{SCH} \mathrm{R}$ & 0 & 8 & .0 & 1.000 \\
\hline
\end{tabular}

ii. Physician-Centered Clinics $(\alpha=30, \beta=30, \omega=1)$

\begin{tabular}{|c|c|c|c|c|}
\hline Source & Sum of Sq & $\mathrm{df}$ & $\mathrm{F}$ & Sig. \\
\hline Corrected Model & $851957338^{a}$ & 119 & 54580.4 & 0.000 \\
\hline Intercept & 3185171431 & 1 & 24282805.7 & 0.000 \\
\hline $\mathrm{DL}$ & 661134041 & 1 & 5040290.6 & 0.000 \\
\hline $\mathrm{Pw}$ & 89781304 & 1 & 684466.1 & 0.000 \\
\hline SEAS & 17226665 & 1 & 131331.0 & 0.000 \\
\hline SCH_R & 26285844 & 2 & 100197.8 & 0.000 \\
\hline ACS_R & 27502127 & 4 & 52417.0 & 0.000 \\
\hline $\mathrm{DL} * \mathrm{PW}$ & 14539201 & 1 & 110842.6 & 0.000 \\
\hline SEAS $* \mathrm{Pw}$ & 5029397 & 1 & 38342.6 & 0.000 \\
\hline $\mathrm{DL} * \mathrm{ACS} \_\mathrm{R}$ & 4310047 & 4 & 8214.6 & 0.000 \\
\hline SEAS * ACS_R & 2073630 & 4 & 3952.2 & 0.000 \\
\hline $\mathrm{DL} * \mathrm{SCH} \mathrm{R}$ & 1127522 & 2 & 4297.9 & 0.000 \\
\hline $\mathrm{Pw} * \mathrm{ACS} \_\mathrm{R}$ & 814985 & 4 & 1553.3 & 0.000 \\
\hline $\mathrm{Pw} * \mathrm{SCH}$ _R & 710336 & 2 & 2707.7 & 0.000 \\
\hline DL $*$ SEAS & 164094 & 1 & 1251.0 & 0.000 \\
\hline ACS_R $* S C H \_R$ & 18295 & 8 & 17.4 & 0.000 \\
\hline SEAS * SCH_R & 6546 & 2 & 25.0 & 0.000 \\
\hline $\mathrm{DL} * \mathrm{SEAS} * \mathrm{PW}$ & 516676 & 1 & 3939.0 & 0.000 \\
\hline SEAS $*$ Pw $*$ ACS_R & 422184 & 4 & 804.7 & 0.000 \\
\hline $\mathrm{Pw} *$ ACS_R $*$ SCH_R & 76170 & 8 & 72.6 & 0.000 \\
\hline $\mathrm{DL} * \mathrm{SEAS} * \mathrm{ACS} \_\mathrm{R}$ & 64403 & 4 & 122.7 & 0.000 \\
\hline $\mathrm{SEAS} * \mathrm{Pw} * \mathrm{SCH} \mathrm{R}$ & 29446 & 2 & 112.2 & 0.000 \\
\hline $\mathrm{DL} *$ ACS_R $*$ SCH_R & 28749 & 8 & 27.4 & 0.000 \\
\hline $\mathrm{DL} * \mathrm{Pw} *$ ACS_R & 17912 & 4 & 34.1 & 0.000 \\
\hline $\mathrm{DL} * \mathrm{SEAS} * \mathrm{SCH} \_\mathrm{R}$ & 6726 & 2 & 25.6 & 0.000 \\
\hline $\mathrm{DL} * \mathrm{Pw} * \mathrm{SCH} \_\mathrm{R}$ & 3722 & 2 & 14.2 & 0.000 \\
\hline $\mathrm{SEAS} * \mathrm{ACS} \mathrm{R} * \mathrm{SCH} \mathrm{R}$ & 2697 & 8 & 2.6 & 0.009 \\
\hline DL * SEAS * Pw * ACS_R & 51615 & 4 & 98.4 & 0.000 \\
\hline $\mathrm{SEAS} * \mathrm{Pw} *$ ACS_R $* \mathrm{SCH} \_\mathrm{R}$ & 7474 & 8 & 7.1 & 0.000 \\
\hline $\mathrm{DL} * \mathrm{SEAS} * \mathrm{Pw} * \mathrm{SCH} \mathrm{R}$ & 2983 & 2 & 11.4 & 0.000 \\
\hline $\mathrm{DL} * \mathrm{Pw} * \mathrm{ACS} \_\mathrm{R} * \mathrm{SCH} \_\mathrm{R}$ & 1344 & 8 & 1.3 & $0.249^{\mathrm{a}}$ \\
\hline $\mathrm{DL} * \mathrm{SEAS} * \mathrm{ACS} \_\mathrm{R} * \mathrm{SCH} \mathrm{R}$ & 999 & 8 & 1.0 & $0.472^{\mathrm{a}}$ \\
\hline $\mathrm{DL} * \mathrm{SEAS} * \mathrm{PW} * \mathrm{ACS} \_\mathrm{R} * \mathrm{SCH} \mathrm{R}$ & 203 & 8 & .2 & $0.992^{a}$ \\
\hline
\end{tabular}

R Squared $=.978$ (Adjusted R Squared $=.999)$

DL: Demand load, Pw: Probability of walk-ins, SEAS: Seasonality; ACS_R: Access rule, SCH_R: Scheduling rule

a Insignificant at $\alpha=0$. 
difficult settings with higher walk-ins, higher demand load and higher seasonality. The two-way interactions between access rules and scheduling rules (ACS_R x SCH_R) are relatively less significant (see Table 6), also confirmed by the parallel shifts the interaction plots shown in Figure 5. This confirms our earlier findings in Section 5.1 indicating that the best access rule may be determined fairly independently of the scheduling rule used (or vice versa).

Overall, the main and two-way interaction effects explain around $96 \%$ and $3 \%$ of variation in total costs, respectively. Although most three-way interactions and some of the four-way interactions are statistically significant, these account for less than 1 percent of the variation, as calculated over the sum of squared values (see Table 6).

\section{CONCLUSIONS AND FUTURE DIRECTIONS}

This paper combines capacity allocation and patient scheduling decisions in the context of seasonal demand, both for scheduled and walk-in patients. The relevant decisions are access rules that determine the daily capacity allocated to walk-ins and scheduled patients, and scheduling rules that determine the specific timing of scheduled arrivals. For that purpose, a fully-integrated simulation is used for evaluating system performance at the macro and micro levels of analyses. The macro model addresses the assignment of patients who call for appointments to the first available day, and the micro model simulates the in-clinic dynamics of each service day, including stochastic arrivals and service times. An advantage of integration is that all important trade-offs are considered in terms of patients' lead times (i.e., indirect wait times), wait times in the clinic (i.e., direct wait times), and clinic overtime.

In our simulation experiments, eighteen appointment systems, that are combinations of six access rules and three scheduling rules, are tested under a variety of clinic settings with different demand load, seasonality levels, and percentage of walk-ins. The access rule with "no seasonal adjustment" that reserves a fixed daily capacity for walk-ins is included as a benchmark. Access rules with seasonal adjustments vary daily capacity for walk-ins, called the 'reservation level' according to the seasonal demand pattern. The performance of best access rules and scheduling rules are evaluated by extending the traditional efficient frontier approach under multiple criteria.

The results show that performance improvements as high as $10 \%$ are achieved by seasonal adjustments to daily capacity compared to the benchmark policy of "no seasonal adjustment". The choice of an optimal appointment system is not easy to generalize, as it depends on the demand 
load, percentage of walk-ins and seasonality level, as well as the cost preference. Some general guidelines are provided for different clinic environments by deriving the characteristics of best access rules and scheduling rules under different scenarios, illustrated for two hypothetical clinics with extreme cost preferences, referred to as patient-centered vs. physician-centered clinics. In general, the higher the percentage of walk-ins and seasonality levels, the higher the benefits of seasonal adjustments. However, demand load and cost preferences have some interfering effects on this relationship. In terms of scheduling rules, the Even rule that leaves open slot positions for walk-ins uniformly throughout the session emerges as the best option under most scenarios. This is followed by the EndEven rule, which is preferred in physician-centered clinics when both walkins and seasonality levels are high. The third rule, namely the End rule, does not perform optimally under the scenarios tested.

Our findings show that when clinics aim for short lead times, this can be achieved by operating at low demand loads. However, further improvements on the in-clinic measures of direct wait times and overtime require that demand uncertainty is also reduced in terms of both seasonality and percentage of walk-ins. For clinics with very high demand load or tight capacity, complementary mechanisms for controlling walk-in rates, such as encouraging patients to schedule appointments and/or stricter admission rules for walk-ins may be necessary.

We conclude that the integration of the capacity allocation and patient scheduling decisions is beneficial in hybrid systems with two streams of seasonal demand, illustrated for scheduled and walk-in patients. We show that the best choice of access rules and scheduling rules change when an integrated model is used, as opposed to when each level is studied separately. A full factorial analysis of variance ANOVA is conducted to explore the main and interaction effects between the environmental factors and decision factors. Under the environments tested, the most critical factors emerge as the demand load, walk-ins and seasonality level, listed in the decreasing order of importance. We find that the total cost performance is more highly affected by the choice of scheduling rules compared to access rules. Furthermore, the decisions on these rules are highly independent of one another. This means that similar access rules are preferable regardless of the choice of a scheduling rule or vice versa.

Our analysis focused on seasonal adjustments using a fixed adjustment factor, $\gamma$ over the entire year. Future extensions may evaluate hybrid policies that consider periodic adjustments with differing $\gamma$-values during low/high levels of seasonal demand. Future work may continue to test 
variations where walk-ins could be delayed or rejected with a penalty cost. Walk-in arrivals may also be modeled more realistically to include balking and reneging behaviors. Other simplifying assumptions, such as single-phase, single-server system, or identical and lognormal service times for walk-ins and scheduled patients, may be relaxed in future extensions of the study. New appointment systems could be tested under a wider range of scenarios, including patient preferences, cancellations, no-shows, and patient unpunctuality. Although investigated in the context of seasonal walk-ins, the integration of macro and micro-levels of analysis may be applied to similar settings, where daily capacity is divided among groups of patients with different characteristics.

\section{ACKNOWLEDGEMENTS}

This research is funded by the Scientific and Technological Research Council of Turkey with TUBITAK 3501 grant 109K451. 


\section{REFERENCES}

1. Ahmadi-Javid, A., Jalali Z., Klassen K. (2017). Outpatient appointment systems in healthcare: A review of optimization studies. European Journal of Operational Research, 258(1), 3-34.

2. Bailey, N. (1952). A study of queues and appointment systems in hospital outpatient departments with special reference to waiting times. Journal of the Royal Statistical Society, 14, 185-199.

3. Balasubramanian, H., Muriel A., Wang, L. (2012). The impact of provider flexibility and capacity allocation on the performance of primary care practices. Flexible Services and Manufacturing Journal, 24(4), 422-447.

4. Borgman, N.J., Vliegen, I.M.H., Boucherie, R.J., Hans, E. (2017). Appointment scheduling with unscheduled arrivals and reprioritization Flexible Services and Manufacturing Journal, doi:10.1007/s10696-016-9268-0.

5. Cayirli, T., Veral, E. (2003). Outpatient scheduling in health care: a review of literature. Production and Operations Management, 12(4), 519-549.

6. Cayirli, T., Yang, K. K., Quek, S.A. (2012). A universal appointment rule in the presence of noshows and walk-ins. Production and Operations Management, 21(4), 682-697.

7. Cayirli, T., Gunes, E. D. (2014). Outpatient appointment scheduling in presence of seasonal walkins. Journal of the Operational Research Society, 65(4), 512-531.

8. Cayirli, T., Yang, K. K. (2014). A universal appointment rule with patient classification for service times, no-shows and walk-ins. Service Science 6(4), 274-295.

9. Creemers, S., Lambrecht, M. (2010). Queueing models for appointment-driven systems. Annals of Operations Research, 178(1), 155-172.

10. Dobson, G., Hasija, S., Pinker, E. J. (2011). Reserving capacity for urgent patients in primary care. Production and Operations Management, 20(3), 456-473.

11. Forjuoh S. N., Averitt, W. M., Cauthen, D. B., Couchman, G. R., Symm, B., Mitchell, M. (2001). Open-access appointment scheduling in family practice: Comparison of prediction grid with actual appointments. The Journal of American Board of Family Practice, 14(4), 259-265.

12. Gupta, D., Denton, B. (2008). Appointment scheduling in health care: Challenges and opportunities. IIE Transactions, 40(9), 800-819.

13. Gupta, D., Wang, L. (2008). Revenue management for a primary-care clinic in the presence of patient choice. Operations Research, 56(3), 576-592.

14. Ho, C. J., Lau, H. S. (1992). Minimizing total cost in scheduling outpatient appointments. Management Science, 38(12), 1750-1764.

15. Klassen, K. J., Rohleder, T. R. (1996). Scheduling outpatient appointments in a dynamic environment. Journal of Operations Management, 14(2), 83-101.

16. Klassen, K. J., Rohleder, T. R. (2004). Outpatient appointment scheduling with urgent clients in a dynamic, multi-period environment. International Journal of Service Industry Management, 15(2), 167-186.

17. Koeleman, P. M., Koole, G. M. (2012). Optimal outpatient appointment scheduling with emergency arrivals and general service times. IIE Transactions on Healthcare Systems Engineering, 2(1), 14-30.

18. Kolisch, R., Sickinger, S. (2008). Providing radiology health care services to stochastic demand of different customer classes. OR Spectrum, 30(2), 375-395.

19. Kopach, R., DeLaurentis, P. C., Lawley, M., Muthuraman, K., Ozsen, L. Rardin, R., Wan, H., Intrevado, P., Qu, X., Willis, D. (2007). Effects of clinical characteristics on successful open access 
scheduling. Health Care Management Science, 10(2), 111-124.

20. Kortbeek, N., Zonderland, M. E., Braaksma, A., Vliegen, I. M. H., Boucherie, R. J., Litvak, N., Hans, E. W. (2014). Designing cyclic appointment schedules for outpatient clinics with scheduled and unscheduled patient arrivals. Performance Evaluation, 80, 5-26.

21. Luo, J., Kulkarni, V. G., Ziya, S. (2015). A Tandem Queueing model for an appointment-based service system. Queueing Systems 79(1) 53-85.

22. Ma, X., Sauré, A., Puterman, M. L., Taylor, M., Tyldesley, S. (2015). Capacity planning and appointment scheduling for new patient oncology consults. Health Care Management Science, 1-15.

23. Morikawa, K., Takahashi, K. (2016). Scheduling appointments for walk-ins. International Journal of Production Economics. 190, 60-66.

24. Murray, M., Tantau, C. (1999). Redefining open access to primary care. Managed Care Quarterly, $7(3), 45-55$.

25. Murray, M. Tantau, C. (2000). Same-day appointments: Exploding the access paradigm. Family Practice Management, 7(8), 45-50.

26. Murray, M., Berwick, D. M. (2003). Advanced access: Reducing waiting and delays in primary care. Journal of the American Medical Association, 289(8), 1035-1040.

27. Patrick, J., Puterman, M. L. (2007). Improving resource utilization for diagnostic services through flexible inpatient scheduling: A method for improving resource utilization. Journal of the Operational Research Society, 58(2), 235-245.

28. Patrick, J., Puterman M. L., Queyranne, M. (2008). Dynamic multipriority patient scheduling for a diagnostic resource. Operations Research, 56(6), 1507-1525

29. Qu X., Peng Y., Shi J., LaGanga, L. (2015). An MDP model for walk-in patient admission management in primary care clinics. International Journal of Production Economics, 168, 303-320.

30. Qu, X., Rardin, R. L., Williams, J. A. S., Willis, D. R. (2007). Matching daily healthcare provider capacity to demand in advanced access scheduling systems. European Journal of Operational Research, 183(2), 812-826.

31. Qu, X., Shi, J. (2009). Effect of two-level provider capacities on the performance of open access clinics. Health Care Management Science, 12(1), 99-114.

32. Qu, X., Shi, J. (2011). Modeling the effect of patient choice on the performance of open access scheduling. International Journal of Production Economics, 129, 314-327.

33. Ratcliffe, A., Gilland, W., Marucheck A. (2012). Revenue management for outpatient appointments: joint capacity control and overbooking with class-dependent no-shows A. Flexible Services and Manufacturing Journal (2012) 24(4), 516-548.

34. Rohleder, T. R., Klassen, K. (2002). Rolling horizon appointment scheduling: A simulation study. Health Care Management Science, 5(3), 201-209.

35. Sauré, A., Patrick, J., Tyldesley, S., Puterman, M. L. (2012). Dynamic multi-appointment patient scheduling for radiation therapy. European Journal of Operational Research, 223(2), 573-584.

36. Wang, W. Y., Gupta, D. (2011). Adaptive appointment systems with patient preferences. Manufacturing \& Service Operations Management, 13(3), 373-389.

37. Wang, S., N. Liu, and G. Wan (2017). Managing appointment-based services in the presence of walkin customers." Working paper. Columbia University.

38. Zacharias, C., Armony, M. (2016). Joint panel sizing and appointment scheduling in outpatient care. Management Science, forthcoming. 


\section{Appendix 1. Formulation of the Demand Process with Seasonality and Demand Load Effects}

In this section we formulate the demand process for walk-ins and scheduled patients, both following the same demand pattern based on a multiplicative seasonality index $I_{t}$ for day $t$. This demand process is used to generate calls and walk-ins in the simulation model.

Daily number of walks-ins and scheduled patients are modelled as Poisson processes with yearly average rates $\lambda_{w}$ and $\lambda_{s}$ respectively. Let $T$ be the available capacity in number of slots per day, and $P_{w}$ be the percentage of walk-ins in the total demand, i.e., $P_{w}=\lambda_{w} /\left(\lambda_{w}+\lambda_{s}\right)$. The average utilization or demand load, $\rho=\left(\lambda_{w}+\lambda_{s}\right) / T$ is assumed to be less than 1 .

In the simulation experiments $T$ is fixed. Environmental parameters are $P_{w}, \rho$ and the seasonality level that determines the seasonality index $I_{t}$. The; impact of these are investigated with a full factorial experimental design. In the following, we derive the demand rates and reservation levels in terms of the environmental factors.

Using the definitions of $\rho$ and $P_{w}$ given above, the daily number of slots $T$ can be written as

$$
T=P_{w} T+\left(1-P_{w}\right) T=\frac{\lambda_{w}}{\rho}+\frac{\lambda_{s}}{\rho}
$$

Using (A1) and multiplying the average rates with the seasonality index, we can write the daily demand rate for walk-ins as:

$$
\lambda_{w}^{t}=\lambda_{w} I_{t}=\rho P_{w} T I_{t}
$$

Similarly, given the overall average call rate for scheduled appointments $\left(\lambda_{s}\right)$, the average arrival rate of calls for day $t$ is calculated as follows:

$$
\lambda_{s}^{t}=\lambda_{s} I_{t}=\rho\left(1-P_{w}\right) T I_{t}
$$

Our simulation model uses $\lambda_{w}^{t}$ and $\lambda_{s}^{t}$ for generating seasonal demand patterns for walk-ins and scheduled patients who call for appointments. However, our access rules define the reservation level on a daily basis based on $\lambda_{w}^{t}$, which is adjusted by coefficient $\gamma$. As a result, the daily reservation level for walk-ins is represented as follows:

$$
R_{t}=P_{w} T I_{t} \gamma=\frac{\lambda_{w}^{t}}{\rho} \gamma=\frac{\lambda_{\mathrm{w}} I_{t}}{\rho} \gamma
$$

After rearranging terms in Equation (A1), the decision variable $\gamma$ may be interpreted through its impact on the daily average booking limit $N_{t}$, represented as follows:

$$
N_{t}=T-R_{t}=\frac{\lambda_{s}}{\rho}+\frac{\lambda_{w}}{\rho}-\frac{\lambda_{\mathrm{w}} I_{t}}{\rho} \gamma=\frac{\lambda_{s}}{\rho}+\lambda_{w} \frac{1-I_{t} \Upsilon}{\rho}
$$

The benchmark yearly average booking limit $N$ in the case when there is no seasonal adjustment can be found by setting $I_{t} \gamma=1$. The second part of A4 represents the change in the booking limit as a result of adjustment for seasonality of walk-ins. When $I_{t} \gamma>1$, the daily booking limit $N_{t}$ is less than the yearly average booking limit $N$, since $\lambda_{w} \frac{1-I_{t} \Upsilon}{\rho}<0$. When $P_{w}$ is high (i.e., $\lambda_{w}$ is high) and seasonality level is "High", $\lambda_{w} I_{t}$ is expected to be very high for some days, and therefore, we expect the best $\gamma$ to decrease in order to alleviate the change on the booking limit. Our numerical examples in Section 5.3 confirm this expectation. It is observed that $\gamma$ is lower in Env_1 (DL $=99 \%, S E A S=$ High, $\left.P_{w}=80 \%\right)$ compared to Env_2 $\left(D L=99 \%, S E A S=\right.$ Low, $\left.P_{w}=40 \%\right)$, and similarly, in Env_3 compared to Env_4 for $D L=80 \%$ (See Table 5). 


\section{Appendix 2. Extended Efficient Frontier Analysis for Trade-offs between Wait Time, Lead}

\section{Time and Overtime}

This section extends the analysis in Section 5 by plotting results on the efficient frontier when all trade-offs are included. For this purpose, patient's lead time and wait time measures are combined into a single measure for a given $\omega$-ratio defined as $c_{W} / c_{L}$. This revises the original total cost equation $T C_{\text {Int }}$ (Equation 3) as follows:

$$
T C_{\text {Int }}=c_{W}\left(L T / \omega+W T_{T}\right)+c_{O}(O T)
$$

Figure A2 presents the efficient frontiers for two values of $\omega=1$ and 5 for the "high" environment with $D L=99 \%, S E A S=\mathrm{H}, P_{w}=80 \%$ (Env_1 in Section 5). Performance of all appointment systems are tested, inluding 18 appointment systems (i.e., combinations of End, Even, EndEven rules with Seas_Adj with $\gamma=0.85-1.00$ and No_SeasAdj). For a given $\omega$, the final choice depends the cost ratio, $\beta$, as indicated by different slopes on the efficient frontiers. We note that choosing two cost ratios $(\omega$ and $\beta)$, automatically sets the third one $(\alpha)$. For example, if $\omega=5, \beta=$ 30 , then $\alpha=150$.

From Figure A2, for a given choice on $\omega$, only a limited set of appointment systems remain on the efficient frontier, while the rest - including the benchmark policies of No_SeasAdj are inferior. The $\beta$-values indicate the range at which each appointment system becomes the best choice. When $\omega=1$, the decision-maker will choose SeasAdj_1.00_End if $\beta \geq 269$, SeasAdj_1.00_EndEven if 95 $\leq \beta<269$, SeasAdj_0.95_EndEven if $26 \leq \beta<95$, SeasAdj_0.95_Even if $17 \leq \beta<26$, SeasAdj_0.90_Even if $5 \leq \beta<17$, and SeasAdj_0.85_Even if $1 \leq \beta<5$. Similarly, when $\omega=5$, the best choice is SeasAdj_1.00_End if $\beta \geq 269$, SeasAdj_1.00_EndEven if $28 \leq \beta<269$, SeasAdj_1.00_Even if $28 \leq \beta<7$, and SeasAdj_0.95_Even if $1 \leq \beta<7$. These results are parallel with those in Section 5.2 (see Figure 3), where $\beta$-values are fixed at 1.5 and 30 for the patient vs. physician-centered clinics.

The complete results on $\beta$-values calculated for $\omega=0.5,1,2,5$ and 10 are included in Table A2. Although 18 appointment systems are included in the analysis, parallel to in Section 5, Table A2 only lists those that appear on the efficient frontiers as the best performing ones for the illustrated Env_1. From Table A2, as $\omega$ increases, smaller sets of appointment systems remain on the efficient frontiers. This is also observed in Figure A2, where there are four best appointment systems for $\omega=$ 5 , as opposed to seven for $\omega=1$. As $\omega$ increases, the frontiers are increasingly dominated by those appointment systems with larger coefficient $\gamma$ (e.g., SeasAdj_1.00). These rules reserve more capacity for walk-ins, and therefore less capacity for scheduled patients, resulting in longer lead times. Thus they are more preferable when $\omega$ and (indirectly) $\alpha$ increase for a given $\beta$, indicating a lower preference for patient lead times compared to direct wait times and overtime.

Table A2. Best Appointment Systems for Different $\omega=0.5,1,2,5,10$

\begin{tabular}{|c|c|c|c|c|c|}
\hline Appointment System & $\omega=0.5$ & $\omega=1$ & $\omega=2$ & $\omega=5$ & $\omega=10$ \\
\hline SeasAdj_1.00_Even & - & - & - & $\geq 7$ & $\geq 1$ \\
\hline SeasAdj_0.95_Even & - & $\geq 17$ & $\geq 3$ & $\geq 1$ & - \\
\hline SeasAdj_0.90_Even & $\geq 22$ & $\geq 5$ & $\geq 1$ & - & - \\
\hline SeasAdj_0.85_Even & $\geq 7$ & $\geq 1$ & - & - & - \\
\hline SeasAdj_0.80_Even & $\geq 1$ & - & - & - & - \\
\hline SeasAdj_1.00_EndEven & $\geq 206$ & $\geq 95$ & $\geq 40$ & $\geq 28$ & $\geq 28$ \\
\hline SeasAdj_0.95_EndEven & $\geq 47$ & $\geq 26$ & $\geq 26$ & - & - \\
\hline SeasAdj_0.90_EndEven & $\geq 26$ & - & - & - & - \\
\hline SeasAdj_1.00_End & $\geq 269$ & $\geq 269$ & $\geq 269$ & $\geq 269$ & $\geq 269$ \\
\hline
\end{tabular}


Figure A2. Efficient Frontiers

i. $\omega=1$

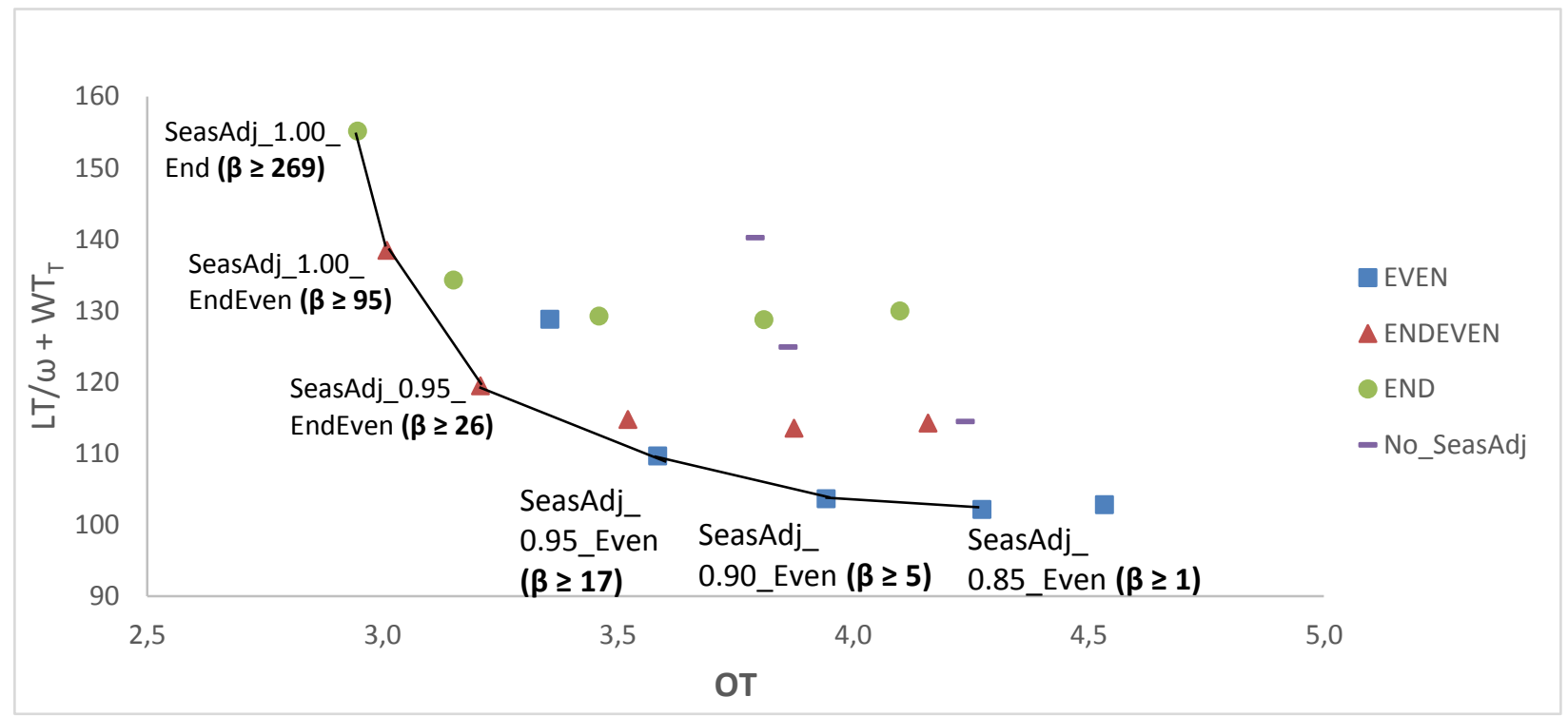

ii. $\omega=5$



